\title{
Progressive Collapse Analysis of Ship Hull Girders Subjected to Extreme Cyclic Bending
}

\section{Shen $\mathrm{Li}^{*}$, Zhiqiang Hu and Simon Benson}

Marine, Offshore and Subsea Technology Group, School of Engineering, Newcastle University, UK

*Corresponding e-mail: s.li37@newcastle.ac.uk

\section{Highlights}

- A novel method is proposed to predict the progressive collapse response of ship hull girders under cyclic bending;

- Application on ship-type box girders using an extreme cyclic loading which surpasses the monotonic ultimate strength;

- Analyses are validated against experimental data and numerical simulations;

- Extreme cycles of load are shown to cause a progressive strength degradation of the box girders.

\begin{abstract}
This paper introduces a novel analytical method to predict the buckling collapse behaviour of a ship hull girder subjected to several cycles of extreme load. This follows the general principles of the established simplified progressive collapse method with an extended capability to re-formulate the load-shortening curve of structural components to account for cyclic degradation. The method provides a framework for assessing residual hull girder strength following a complex series of unusually extreme load events where the wave induced bending moment rises close to, or even surpasses, the monotonic ultimate strength. These load events may be sequential, such as might be caused by a series of storm waves, or they may occur as a collection of discrete events occurring over a longer period. The extreme cyclic bending amplifies the distortion and residual stress initially induced by fabrication in the flanges of the girder, which results in a deterioration of the residual ultimate strength. Validation is firstly completed through a comparison with previously published experimental work and secondly via comparison with numerical simulation on four ship-type box girders using the nonlinear finite element method.
\end{abstract}

Keywords: Ultimate strength; buckling; longitudinal strength; ship hull girder; cyclic load; progressive collapse. 


\section{Introduction}

Ultimate limit state assessment is now the preferred approach within statutory design guidance for determining the global strength of a ship [1-2]. This requires a robust prediction of the ultimate strength of the hull girder, which usually follows the simplified progressive collapse method originally introduced by Smith [3]. This method has been experimentally validated, for example by Dow [4], and is now codified in classification society rules for ships, such as [1-2].

The ship hull girder strength assessment using the original Smith method assumes monotonic loading. However, in the unusual event of an overall failure of a hull girder due to bending, the collapse is likely to have been driven by a complex series of extreme wave load events which load the girder cyclically. Such cyclic load events may occur sequentially over a short period, such as might be caused by a series of storm waves. Alternatively, they may occur as a collection of discrete load events occurring over a much longer period stretching over the lifespan of the vessel, with each event causing progressive degradation of the hull girder strength leading to a corresponding reduction in the safety margin.

Although most ultimate strength failures of ships are considered as distinct single events, their causes may be rooted in accumulative elastoplastic buckling degradation within highly loaded areas of the hull structure until the ultimate strength is surpassed and the hull girder fails. In these scenarios, the magnitude of the load cycles contributing to the degradation may be significantly less than the nominal ultimate hull girder strength. For instance, in the failure analysis of an inland waterway oil bunker barge, the collapse was largely attributed to progressive damage, which resulted in inelastic behaviours during repeated cycles of loading [5]. In the aftermath of the MOL Comfort disaster, a repeated load between the still water bending moment and $90 \%$ of the ultimate bending moment was applied to verify the buckled bottom plating found on the sister ships [6]. An accumulatively increased distortion was reproduced, which may have had an adverse effect on the ultimate ship hull strength performance.

The cyclic loading may continue to act on a ship hull even after the ultimate hull girder strength is exceeded, in which case the response may also surpass the nominal ultimate capacity. This series of extreme cyclic loading may eventually result in the destructive final event, with fracture induced in the highly loaded panels that leads to the hull girder "breaking its back". Within this context, lijima and Fujikubo experimentally investigated the cumulative damage and collapse extent of a small-scale ship model under a series of extreme waves [7]. A hydro-elastoplasticity method was proposed to simulate the post-ultimate strength behaviour of a ship where both the fluid-structural coupling and nonlinear structural stiffness are accounted for [8]. In this methodology, the nonlinear structural stiffness was approximated either by piece-wise linear curves or a series of spline curves which smoothly interpolate the measured points in a static test. The unloading stiffness was taken as equal to the initial stiffness and the reloading response was assumed to follow the same path. The study on the post-ultimate strength behaviour would provide an enhanced risk assessment associated with the hull girder collapse. 
Cyclic loading therefore has important implications for the safety and survivability of the ship. Both 14th and 15th International Ship and Offshore Structures Congress (ISSC) highlighted the consequences of cyclic failure in ship hull girders [9-10]. They concluded that the residual structural capacity after several cycles of extreme loading can be significantly lower than the instantaneous collapse strength under a single load excursion, since the reversal and accumulation of plastic deformation permanently degraded the structural resistance against subsequent loading.

The few studies concerning the cyclic response of ship-type structural components also show a degradation in ultimate capacity after several cycles of load. A series of finite element analyses (FEA) on unstiffened plates under in-plane cyclic tension and compression was conducted in [11] Stemming from observations in the numerical study, Yao et al. proposed an analytical method to predict the cyclic response of plates and stiffened panels based on elastic large deflection and rigid-plastic mechanism theory [12]. Fukumoto and Kusama experimentally and analytically studied the behaviour of a thinwalled beam under cyclic bending [13]. An experimental programme was carried out by Kaminski to investigate the load-carrying capacity of a series of imperfect plates being cyclically compressed [14]. Recently, an experimental test was reported to investigate the ultimate strength and failure characteristics of a steel box girder under cyclic bending moment [15]

Overall, the number of investigations on cyclic loading within the research area of ship structure ultimate strength are very limited in open literature compared to monotonic loading. No theoretical methodology has so far been proposed to predict the cyclic bending response of a stiffened plating hull girder structure. The nonlinear finite element method (NLFEM) may be used to perform such simulations, but the drawbacks of high computational cost and uncertainty caused by different finite element solvers, setup and parameters are considerable.

This paper aims to extend the capability of the simplified progressive collapse method to predict the response of ship hull girders under cyclic load, which is hereafter denoted as the cyclic progressive collapse method. To confirm the validity of the proposed methodology, the present study is confined to the simulation of a series of unusually extreme loading cycles that surpass the monotonic ultimate strength. The proposed cyclic progressive collapse method follows the assumptions and overall procedure of the original Smith method. The prediction of cyclic bending response is attributed to the re-formulation of component load-shortening curves. The proposed algorithm is described and validations are completed through comparison with experimental data and nonlinear FEA. Furthermore, the apparent strength reduction of ship hull girders caused by multi-cycle loads is investigated. The uncertainty of prediction is discussed and a recommendation for further study is suggested. 


\section{Background}

\subsection{Development of the Simplified Progressive Collapse Method}

Caldwell proposed the first method to predict the ultimate bending strength of ship hull girders, which was based on a presumed stress distribution of the hull girder cross section at the collapse state [16]. However, Caldwell's method was unable to consider the progressive collapse behaviour of a hull girder cross section. Smith resolved this issue with the introduction of the simplified progressive collapse method [3]. Similar approach was also developed in [17-21]. The original Smith method was extended to deal with the combined vertical and horizontal bending problem in [22]. Benson et al. continued to extend the original method by removing the interframe collapse assumption [23] and Syrigou et al. extended its capability to introduce combined vertical and torsional bending [24]. While the simplified progressive collapse method can be applied to analyse the collapse behaviour of intact hull girders, applications on damaged ships were performed by Fujikubo et al. [25] and Dow [26]. In the same time, a large amount of developments focused on improving the estimation of component load-shortening curves. A specialised finite element method was introduced in [27]. An approximate method accounting for beam-column buckling and stiffener tripping was described in [28]. More recently, a load-shortening curve library based on nonlinear FEA was developed in [29].

Benchmark studies on the Smith method have been regularly conducted by the Ultimate Strength Committee of ISSC. Whist there are some uncertainties primarily caused by different formulations of load-shortening curves, the simplified progressive collapse method has proved an efficient and reliable methodology for the evaluation of longitudinal strength of ship structures. The simplified progressive collapse method is thereby incorporated in the Common Structural Rules (CSR) issued by the International Association of Classification Society [1-2].

In parallel to the development of Smith-type simplified progressive collapse method, an idealised structural unit method (ISUM) was proposed by Ueda and Rashed [30]. ISUM follows the general framework of the NLFEM, but allows for the use of larger element size and therefore increase the computational efficiency, which is attributed to the simplification of the evaluation of geometric and material nonlinearity. The formulations underpinning these large elements are quite equivalent to the load-shortening curves used within the standard Smith method. Therefore, the research and subsequent extension to the Smith method proposed in this paper may have an implication on the development of ISUM element to simulate the collapse behaviour under cyclic load.

\subsection{Assumptions and Procedure of the Simplified Progressive Collapse Method}

The following assumptions are made in the simplified progressive collapse method:

- Simple beam assumption: The cross section remains plane during the progressive collapse;

- Independency assumption: Interaction does not exist between the adjacent elements; 
- Interframe collapse assumption: Transverse frames are sufficiently strong so that all of the elements fail in an inter-frame mode.

The first assumption holds until large deflection and large local deformation are produced. The second assumption may be reasonable when the cross section is mainly subjected to vertical bending [31]. The last assumption is only applicable to conventional ship structural design and has been addressed through an extension to the original Smith method accounting for overall grillage instability that likely occurs in a lightweight ship structure [23].

With these assumptions, an incremental relationship between the bending moment and curvature can be given as Equation (1) with terms as defined in Equations (2) to (7).

$$
\begin{aligned}
& \left\{\begin{array}{l}
\Delta M_{H} \\
\Delta M_{V}
\end{array}\right\}=\left[\begin{array}{ll}
D_{H H} & D_{H V} \\
D_{V H} & D_{V V}
\end{array}\right]\left\{\begin{array}{l}
\Delta \chi_{H} \\
\Delta \chi_{V}
\end{array}\right\} \\
& D_{H H}=\sum_{i=1}^{n} k_{i} A_{i}\left(y_{i}-y_{G}\right)^{2} \\
& D_{V V}=\sum_{i=1}^{n} k_{i} A_{i}\left(z_{i}-z_{G}\right)^{2} \\
& D_{H V}=D_{V H}=\sum_{i=1}^{n} k_{i} A_{i}\left(y_{i}-y_{G}\right)\left(z_{i}-z_{G}\right) \\
& y_{G}=\left(\sum_{i=1}^{n} y_{i} k_{i} A_{i}\right) /\left(\sum_{i=1}^{n} k_{i} A_{i}\right) \\
& z_{G}=\left(\sum_{i=1}^{n} z_{i} k_{i} A_{i}\right) /\left(\sum_{i=1}^{n} k_{i} A_{i}\right) \\
& \Delta \varepsilon_{i}=\left(y_{i}-y_{G}\right) \Delta \chi_{H}+\left(z_{i}-z_{G}\right) \Delta \chi_{V}
\end{aligned}
$$

To implement this incremental approach, the following procedure can be used:

1. The ship hull cross section is sub-divided into structural elements;

2. A load-shortening curve (LSC) characterising the response under in-plane load is assigned to each element;

3. Evaluate the tangent stiffness $k_{i}$ of each element at present strain using the load-shortening curve;

4. Calculate the position of instantaneous neutral axis $\left(y_{G}, z_{G}\right)$ using Equation (5) and (6);

5. Evaluate the bending stiffness of the cross section with respect to the instantaneous neutral axis using Equation (2) to (4);

6. Apply the curvature increment about the instantaneous neutral axis;

7. Calculate the strain increment of each element using Equation (7);

8. Calculate the bending moment increment using Equation (1); 
9. Obtain the cumulative bending moment and curvature;

10. Return to step 3.

Since the ship structure is essentially a network of plates and stiffened panels, there are generally two ways to subdivide the cross section as illustrated in Figure 1. Smith originally adopted the plate-stiffener combination where the stiffener and the attached plate is taken as one element [3]. Alternatively, the local plating between the longitudinal stiffeners or individual stiffener can be considered as one element, which is called the plate-stiffener separation subdivision. After the subdivision of the cross section, an average stress-average strain relationship (load-shortening curve) is assigned to each of these elements. This relationship characterises the structural response of an element under in-plane compression/tension, which is the primary loading arising from the global longitudinal bending of hull girder. The effect of secondary loadings, such as lateral pressure and shear force, can be incorporated by modifying the load shortening curves. The plate-stiffener combination is adopted in the present study, as most of the established methods for the prediction of structural component response are developed based on plate-stiffener combination model, such as CSR method.

\section{A continuous stiffened panel}

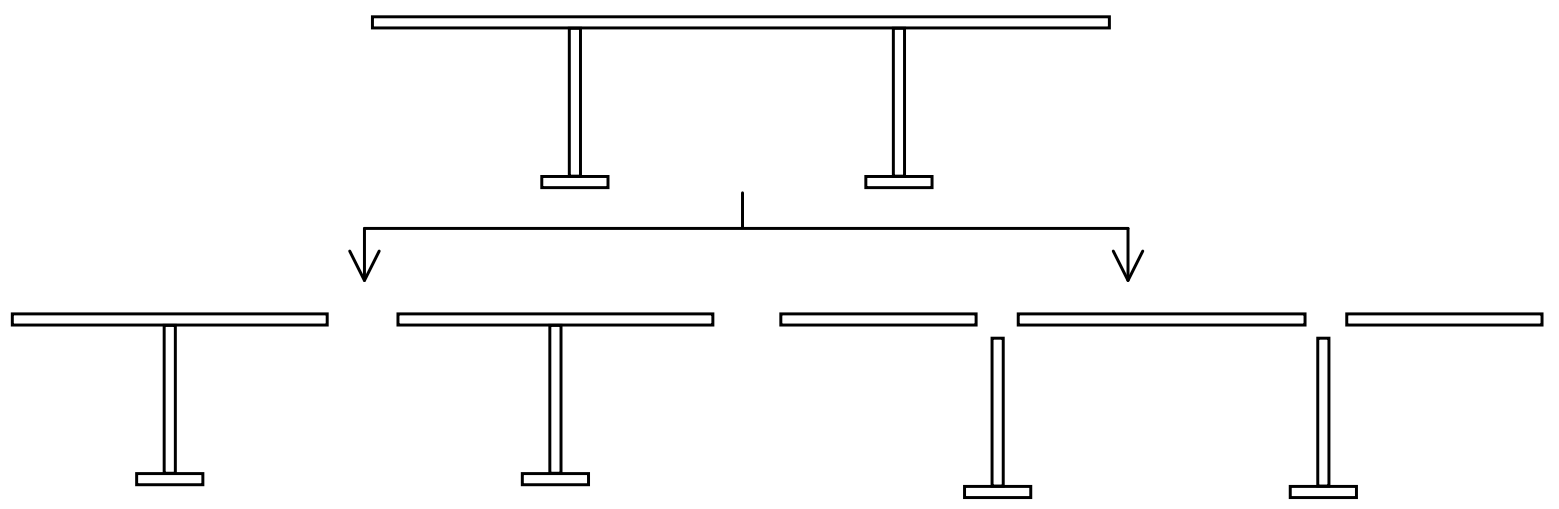

Plate-stiffener combination

Plate-stiffener separation

Figure 1. Subdivision techniques for cross section

\subsection{NLFEM for Collapse Analysis of Ship Structures}

Since the first application by Chen et al [32], the NLFEM has become a commonly used approach to analyse the collapse behaviour of ship structures. For example, the NLFEM was applied to analyse the progressive collapse of bulk carrier in alternate hold loading condition in [33]. The investigation on the MOL Comfort accident was conducted by applying NLFEM to predict the ultimate collapse strength. A concise introduction to the NLFEM and approach for ultimate strength assessment is given in [34]. Practical finite element modelling techniques for ship structures can be found in [35]. 


\section{The Proposed Cyclic Progressive Collapse Method}

\subsection{Principle}

The proposed cyclic progressive collapse method continues to follow the major assumptions and procedure of the simplified progressive collapse method described in Section 2.2, but re-formulates the load-shortening curve (LSC) to account for the accumulated degradation during cycles of extreme load. The overall methodology can be described with reference to Figure 2, in which the extension to the original Smith method is highlighted. The re-formulation of the LSC is driven by a loading protocol which allows the direction of each curvature increment to be controlled. When the direction of curvature increment is reversed, the re-formulation algorithm will be activated. This updated LSC will be utilised for the subsequent Smith method calculations until the next curvature increment reversal.

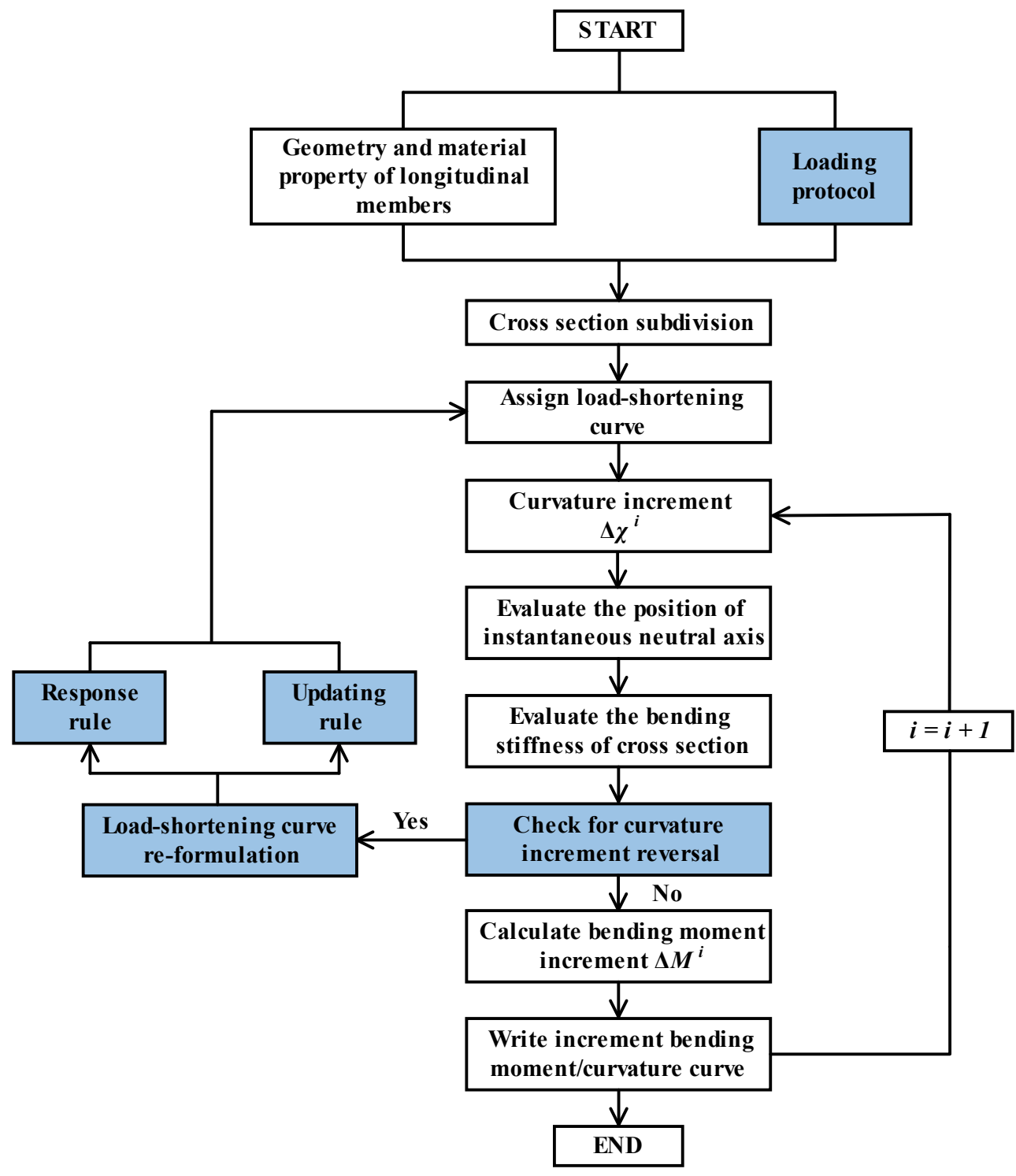

Figure 2. Flowchart of the cyclic progressive collapse method 


\subsection{Load-Shortening Response of Structural Components under Cyclic Load}

To predict the load-shortening response of structural components under cyclic load, an analytical method was derived in [36]. Based on the observed response patterns from a parametric nonlinear finite element analysis (FEA) on unstiffened plates under cyclic compression and tension, a response rule and updating rule were proposed which enabled the prediction of a new response path after a reversal in direction. Validation was completed on unstiffened plates and stiffened panels in [39], proving that the analytical method is valid for both structural components. A concise introduction to the methodology introduced in [36] is given in the following.

Figure 3 shows the typical load-shortening responses of a stiffened panel under cyclic loading, which are predicted by dynamic implicit FEA solver with consideration of Chaboche material hardening (Table 1). The general behaviours of the stiffened panel under cyclic loading are the same as the observations on unstiffened plates investigated in [36]. When the initial loading is applied in compression, the response path of reloading to tension exhibits a considerably lower stiffness compared with the monotonic tensile response (Figure 3a). Although a distinct tensile ultimate limit point may not exist, it is clear that an obvious transition into the nonlinear response region, marked by a drastic decrease of the stiffness, takes place approximately at $\varepsilon / \varepsilon_{Y e q}=-1$. By contrast, if tensile loading has previously been applied (Figure $3 b$ ), the reloading path to tension tends to approach the previous tensile unloading point, resulting in a much lower in-plane stiffness. A significant strength reduction can be found during the second reloading in compression (Figure 3a). Further, the stiffness in post-collapse regime becomes smaller, suggesting a more gradual drop of the post-ultimate strength. On the other hand, when the initial loading is applied in tension, the response path of reloading to compression is close to the behaviour of monotonic compression regarding to stiffness and strength (Figure 3b). As for the second reloading in tension, the tensile strength is slightly reduced aside from a much lower stiffness as introduced before. The strength reduction in both compression and tension during the cyclic load may be interpreted as caused by the amplified distortion and residual stress, which are induced by the elastoplastic buckling experienced in the previous compressive load. This may effectively alter the "initial condition" for the subsequent load cycle and thus leads to a strength reduction. Regarding to multi-cycle loading, it can be noted from Figure 4 that the post-ultimate strength stiffness in compression tends to zero after three loading cycles, and the reduction of compressive strength levels off. Conversely, the tensile strength keeps reducing with a nearly constant reduction rate. 


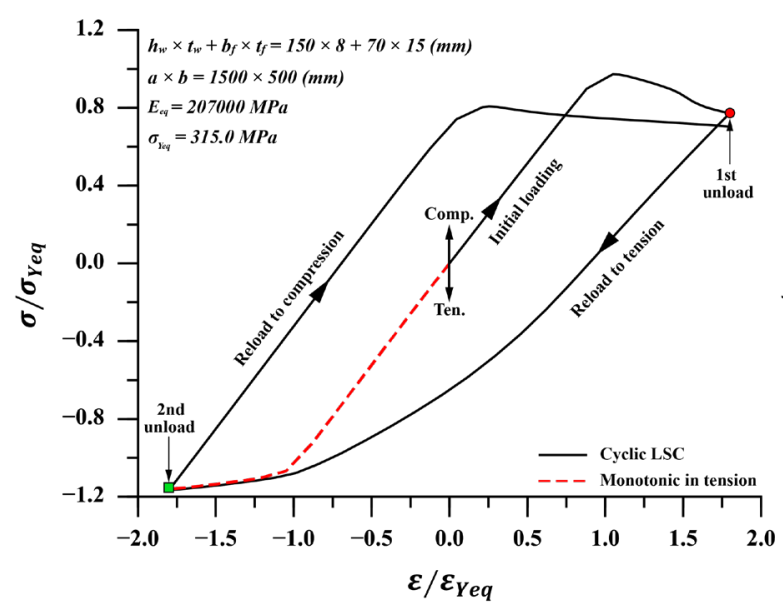

(a)

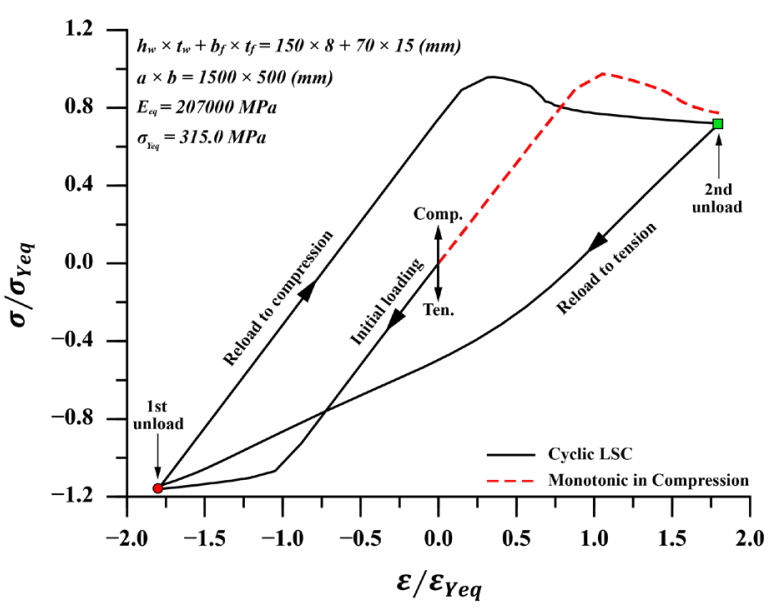

(b)

Figure 3. Typical response pattern of stiffened panel under cyclic loading (Nonlinear FEA)

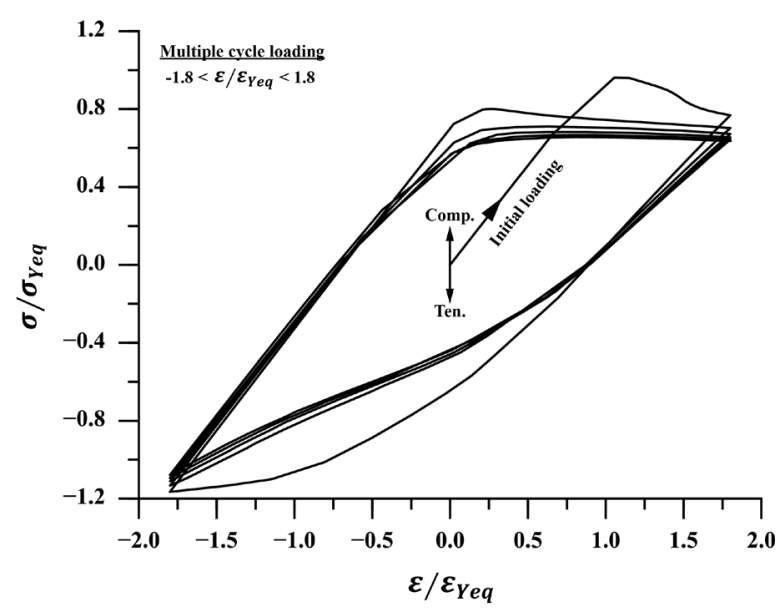

(a)

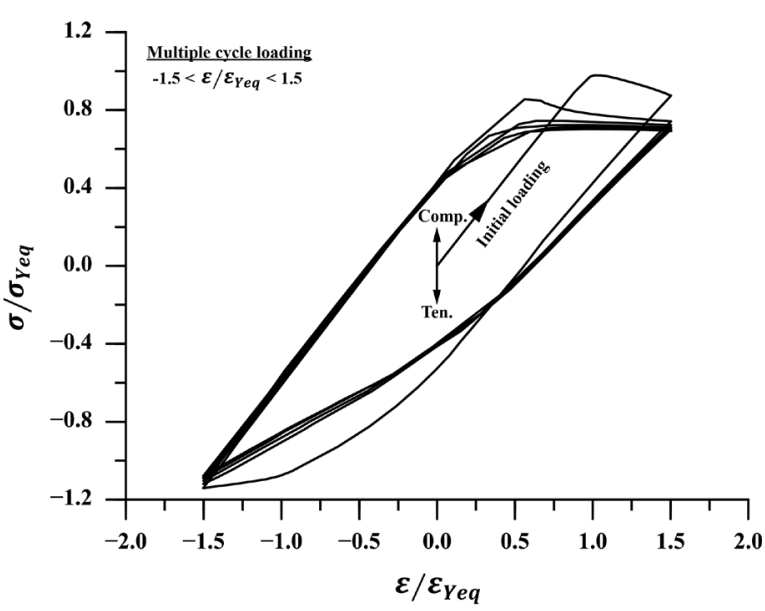

(b)

Figure 4. Typical response pattern of stiffened panel under multi-cycle loading (Nonlinear FEA)

Table 1. Parameters of the Chaboche cyclic plasticity material model [37]

\begin{tabular}{|c|c|c|c|c|c|c|}
\hline Elastic behaviour & \multicolumn{2}{|c|}{$\begin{array}{l}E \text { E (MPa) } \\
207000\end{array}$} & \multicolumn{2}{|c|}{$\begin{array}{c}v \\
0.3\end{array}$} & \multicolumn{2}{|c|}{$\begin{array}{c}\sigma_{y}(\mathrm{MPa}) \\
315\end{array}$} \\
\hline \multirow{4}{*}{ Plastic behaviour } & \multicolumn{6}{|c|}{ Kinematic hardening } \\
\hline & $\begin{array}{c}C_{1}(\mathrm{MPa}) \\
13921\end{array}$ & $\begin{array}{c}\gamma_{1} \\
765\end{array}$ & $\begin{array}{c}C_{2}(\mathrm{MPa}) \\
4240\end{array}$ & $\begin{array}{l}\gamma_{2} \\
52\end{array}$ & $\begin{array}{c}C_{3}(\mathrm{MPa}) \\
1573\end{array}$ & $\begin{array}{l}\gamma_{3} \\
14\end{array}$ \\
\hline & \multicolumn{6}{|c|}{ Isotropic hardening } \\
\hline & & $\begin{array}{l}\infty \text { (MF } \\
25.6\end{array}$ & & & $\begin{array}{l}\mathrm{b} \\
4.4\end{array}$ & \\
\hline
\end{tabular}

The response rule holds several critical points that define the overall shape of the load-shortening curve. For each strain increment calculated from the progressive collapse analysis, the response rule is called to calculate the stress increment on the element adhering to the critical points. When the direction of load increment is reversed, the updating rule is activated to modify the critical points held in the 
response rule. This effectively re-formulates the load-shortening curve. The new critical points are utilised in the response rule for the following prediction until the next reversal of load increment.

\subsubsection{Response Rule}

As illustrated in Figure 5, the following critical points are defined in the response rule:

- The permanent deformation point, at which the resultant stress is zero, i.e. Point $b$ in Figure 5;

- The compressive and tensile unloading point, at which the unloading is started;

- The compressive and tensile ultimate point, at which the ultimate strength is attained, i.e. Point a \& c in Figure 5 respectively;

The response rule follows a different approach for compression and tension:

\section{Compression:}

1. The initial compressive response can be predicted by any established method applicable for monotonic loading, such as CSR method and nonlinear FEA;

2. The updated compressive response is idealised as piece-wise linear:

a. The pre-ultimate strength response path follows 'permanent deformation point $\rightarrow$ compressive ultimate point';

b. The post-ultimate strength response follows a constant stiffness determined in the previous update activated by compressive unloading.

\section{Tension:}

1. The tensile response is always assumed as piece-wise linear;

a. If unloading has not taken place in tension, the pre-ultimate strength response path follows 'permanent deformation point $\rightarrow$ tensile ultimate point':

b. If unloading has previously taken place in tension, the pre-ultimate strength response follows the path 'permanent deformation point $\rightarrow$ tensile unloading point $\rightarrow$ tensile ultimate point';

2. The tensile ultimate stress and strain are initialised as the material yield stress and yield strain respectively;

3. In the post-ultimate strength range, the tensile stiffness is taken as zero. 


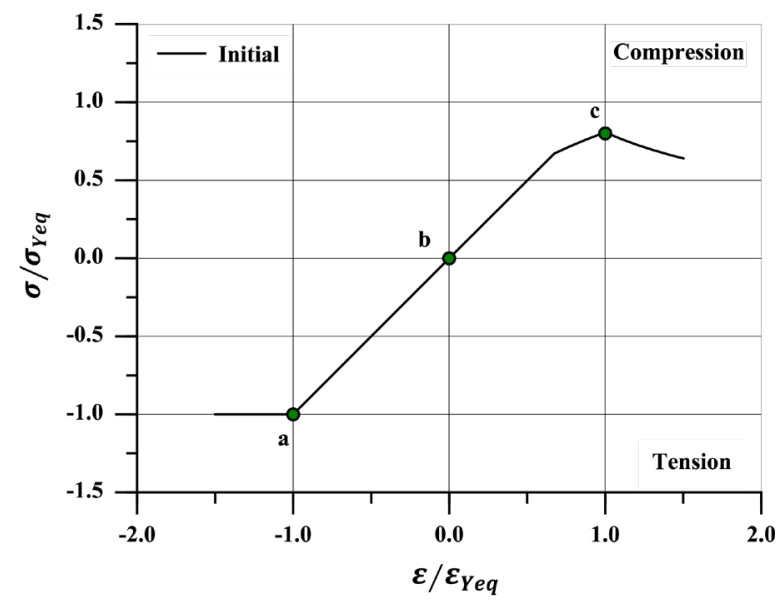

Figure 5. Illustration of the critical points held in the response rule

\subsubsection{Updating Rule}

When a load reversal is initiated, the updating rule will be activated. The last applied strain and resultant stress on the element is recorded as the unloading strain and stress respectively. The following describes the update of relevant data held in the response rule:

1. The stiffness of compressive unloading is estimated as $k=\left(\sigma_{u} / \sigma_{Y e q}\right) \cdot E$ where $\sigma_{u}$ is the initial ultimate compressive strength, $\sigma_{Y e q}$ is the equivalent material yield stress and $E$ is the material Young's modulus;

2. The tensile unloading stiffness is taken as the material Young's modulus;

3. Update the permanent deformation point based on corresponding unloading stiffness;

4. For the unloading in compression, it is assumed that the permanent deformation point remains the same if the unloading strain does not exceed the ultimate strain;

5. If permanent deformation point is unchanged, the update is terminated and all of the data remain the same;

6. If unloading takes place in compression, the post-ultimate stiffness of compression $k_{\text {post }}$ will be updated as $k_{\text {post }}=\varphi_{1}\left(\sigma_{u}-\sigma_{\text {unload }}\right) /\left(\varepsilon_{u}-\varepsilon_{\text {unload }}\right)$ where $\varphi_{1}$ is the compressive post-ultimate strength stiffness reduction factor;

7. If the unloading takes place beyond the ultimate point, the ultimate point is updated as the unloading point;

8. If the unloading takes place at compression, the tensile ultimate strain should be updated in accordance with the tensile strength reduction factor $\varphi_{2}$ to cover the effects of the distortion and residual stress amplified by the previous loading in compression;

9. If the unloading takes place at tension, the critical points relevant to compression remain the same except for the compressive ultimate strain, which should be updated considering the change of the permanent deformation point.

To enable the modelling of compressive strength convergence and tensile strength reduction as found in the nonlinear FEA investigation, the use of reduction factors $\varphi_{1}$ and $\varphi_{2}$ are proposed in updating rule 
6 and 8. The former was specified as 0.3 and the latter was specified as 0.98 in [36]. The validation in [36] on plates and stiffened panels with emphasis on the reloading strength comparison showed a reasonable agreement with equivalent nonlinear FEA. However, as demonstrated in Figure 6 which shows the load-shortening responses of a stiffened panel under different cyclic loadings, it is more sensible to modify the reduction factor $\varphi_{1}$ as a function of the ultimate strain $\varepsilon_{u}$ and unloading strain $\varepsilon_{\text {unload }}$. When the unloading in compression takes place far beyond the ultimate limit point, a considerable change of post-ultimate strength stiffness may occur and the factor $\varphi_{1}$ can follow the recommendation in [36]. By contrast, when the unloading takes place close to the onset of ultimate collapse, the reduction of post-collapse stiffness is less significant. Hence, a linear variation relationship is introduced to accommodate the difference led by the different unloading strain (Equation 8). Overall, the post-collapse compressive stiffness reduction factor $\varphi_{1}$ is specified according to Equation (8) to (10), while the tensile strength reduction factor $\varphi_{2}$ is specified as 0.98 following [36].

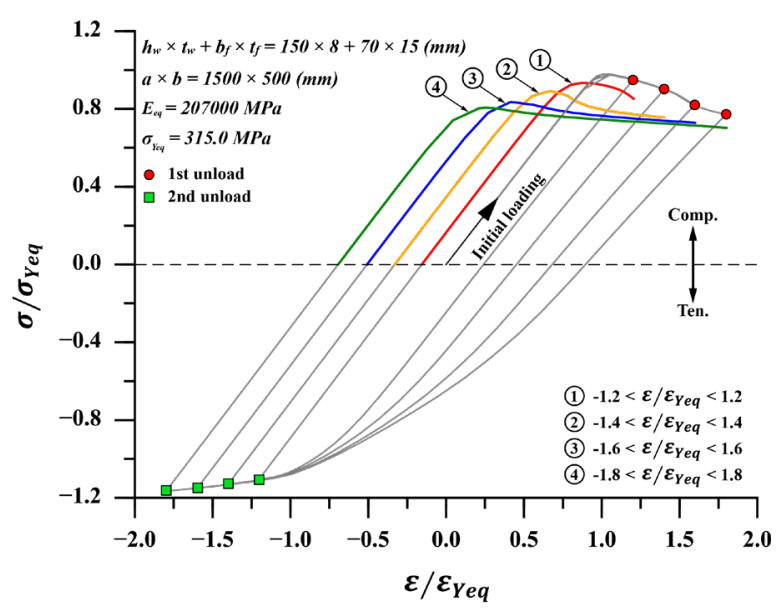

(a)

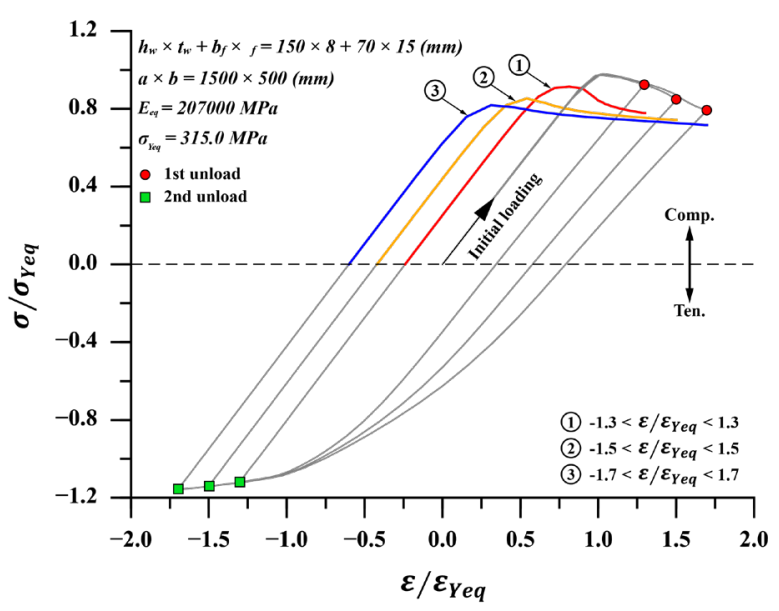

(b)

Figure 6. The effect of different cyclic loading on the post-ultimate strength stiffness in compression

$$
\begin{array}{ll}
\varphi_{1}=1-1.4 \times \bar{\varepsilon} & \text { for } \quad \bar{\varepsilon}<0.5 \\
\varphi_{1}=0.3 & \text { for } \quad \bar{\varepsilon} \geq 0.5 \\
\bar{\varepsilon}=\left(\varepsilon_{\text {unload }}-\varepsilon_{u}\right) / \varepsilon_{\text {Yeq }} &
\end{array}
$$

\subsubsection{Schematic Examples}

The procedure of the response rule and updating rule described above are schematically illustrated in Figure 7 and Figure 8 . The former shows the response initialised by compressive load, while the latter shows the response initialised by tensile load.

As shown in Figure 7(a) where the unloading takes place in compression at Point $e$, the permanent deformation point is calculated as Point $g$ assuming that the compressive unloading stiffness is taken as the slope between Point $c$ and $d$, as stated in updating rule 1 . Since the permanent deformation point 
has changed, the relevant critical points should be updated and the compressive response will be idealised as piece-wise linear according to the compressive response rule 2 . The compressive postultimate strength stiffness (slope $e-f$ ) is taken as a portion of the slope $d$-e, following updating rule 6 . Since there is no tension being applied previously, the critical points relevant to tension remain the same and the tensile pre-ultimate response follows the path 'permanent deformation point $\rightarrow$ tensile ultimate point' (path $g-b$ ) as stated in the tensile response rule $1(a)$. Overall, the LSC is updated as a$b$-g-e-f.

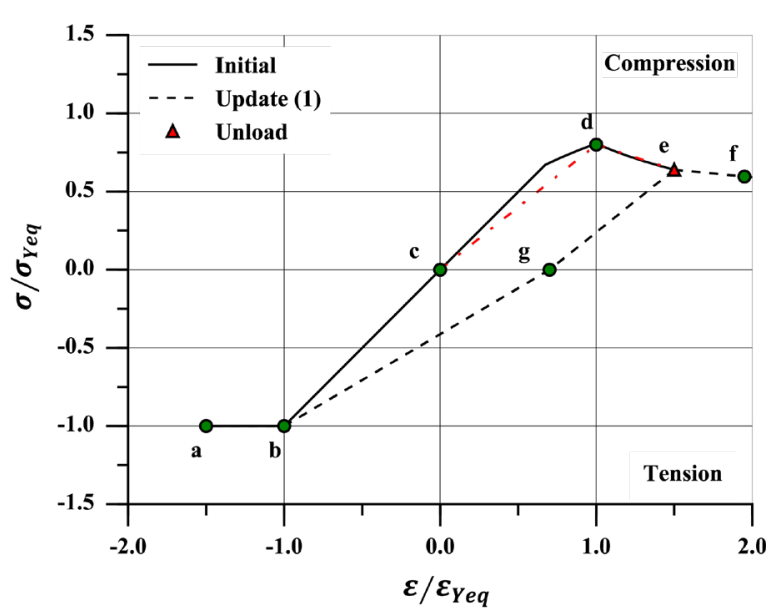

(a)

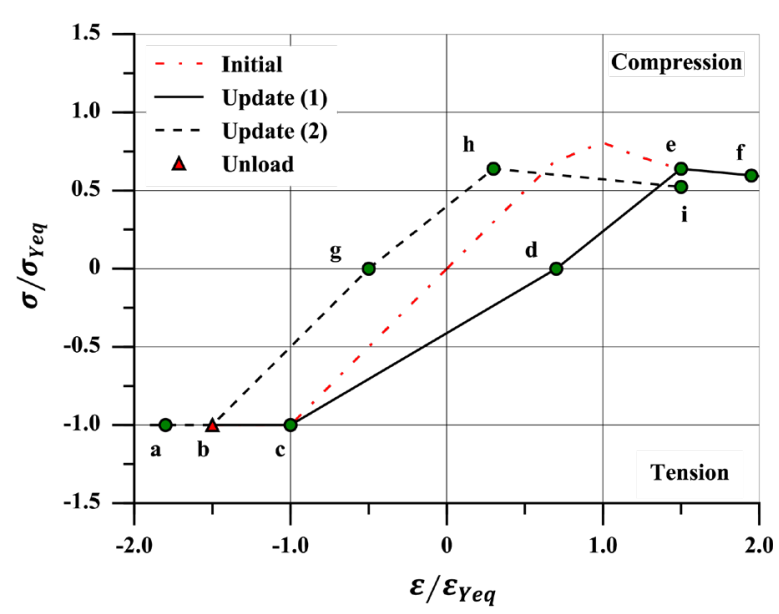

(b)

Figure 7. Schematic example of the response rule and updating rule (Initial loading: compression)

In Figure 8(a) where the unloading takes place in tension at Point $b$, the permanent deformation point is calculated as Point $g$ assuming that the tensile unloading stiffness is taken as the Young's modulus of the material. The tensile ultimate point is updated as Point $b$ and the tensile response follows the path 'permanent point $\rightarrow$ tensile unloading point $\rightarrow$ tensile ultimate point' in accordance with the tensile response rule 1 (b). Since there is no compression unloading taken place previously, the compressive response follows the initially assigned LSC, which can be derived by any established method. However, it is modified to account for the change of the permanent deformation point. Hence, the compressive LSC is shifted from $d-e-f$ to $g-h-i$. Overall, the LSC is updated as $a-b-g-h-i$. A similar procedure is executed in Figure 7(b).

In Figure $8(\mathrm{~b})$ where the unloading takes place in compression at Point $e$, the updating procedure is similar to Figure 7(a) except for the reduction of tensile unloading stress and the change of the tensile ultimate strain according to the updating rule 8 . The updated tensile ultimate strain is determined assuming that the slope of a-h equals to the slope of $h-g$. Overall, the LSC is updated as a-h-g-e-f. 


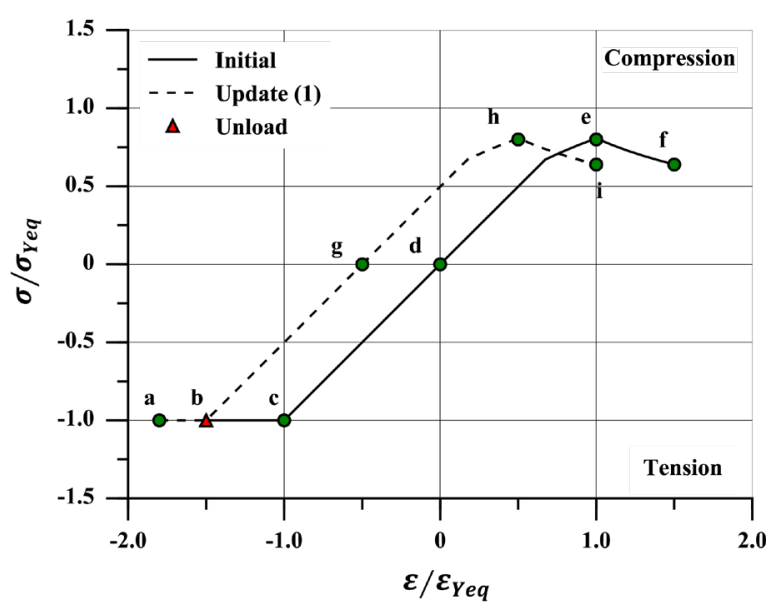

(a)

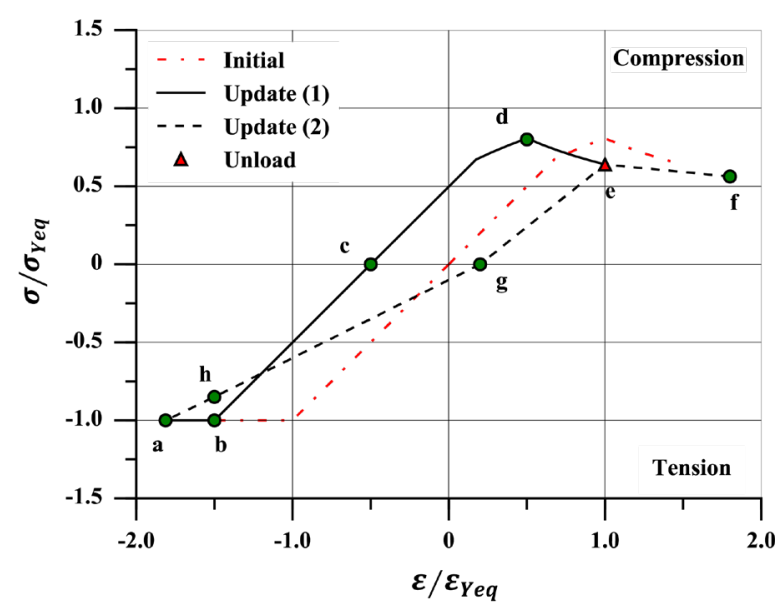

(b)

Figure 8. Schematic example of the response rule and updating rule (Initial loading: tension)

\subsection{Loading Protocol}

The progressive collapse method either curvature or bending moment incrementation. However, the load must be defined in terms of curvature if the cycle extends beyond the ultimate strength [26]. In this study a simple cyclic loading protocol with the same magnitude in both initial and reversed directions is applied for all numerical simulations. The protocol is chosen to demonstrate the validity of the proposed method (e.g. Figure 9) causing an unusually extreme load which surpasses the monotonic ultimate strength of the case-study girders detailed in Section 4. In a more realistic scenario, the purpose of this loading protocol is to represent a complex series of extreme load events in an efficient way for numerical simulations. The loading cycles may occur as a collection of discrete events occurring over a longer period. The loading protocol effectively compresses these events into a sequential format. Completing analyses with a more realistic loading protocol requires further development on integrating the method with a load prediction tool. This is discussed further in Section 6 .

The scope of this study is focused the simulation of a series of extreme loading cycles that surpass the monotonic ultimate strength in order to confirm the validity of the proposed methodology. The loading protocol induces unusually large bending moments on the case study box girders that exceed the nominal ultimate strength. These represent more severe scenarios than would be predicted by design guidelines such as the CSR. The insights developed in this study associated with the implications for ship structures should be taken within the scope of this choice of load protocol. The implications and extension of these findings to normal operational loading conditions requires further research, as summarised in Section 6. 


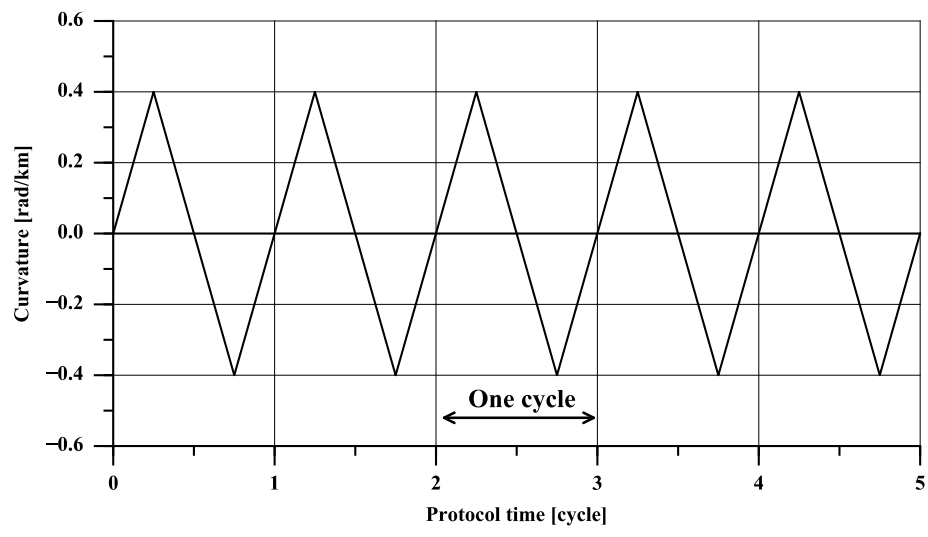

Figure 9. Cyclic loading protocol of ship hull girders in terms of applied curvature

\section{Validation}

\subsection{Experimental Model Characteristics}

The test result reported in [15] is utilised for the experimental validation. The experiment was performed on a small-scaled single-skin box girder model under alternating sagging and hogging. The tested section is $497.9 \mathrm{~mm}$ long with a plating thickness of $2.76 \mathrm{~mm}$. Flat-bar stiffeners with a web height of $50 \mathrm{~mm}$ and thickness of $3.78 \mathrm{~mm}$ are employed. The cross section of the experimental model is shown in Figure 10 with scantlings and material properties listed in Table 2.

Table 2. Dimension and material property of the experimental model [18]

\begin{tabular}{ccccc}
\hline \multirow{2}{*}{ Geometry } & $a[\mathrm{~mm}]$ & $t_{p}[\mathrm{~mm}]$ & $h_{w}[\mathrm{~mm}]$ & $t_{w}[\mathrm{~mm}]$ \\
& 497.8 & 2.76 & 50.0 & 3.78 \\
\hline \multirow{2}{*}{ Material } & $E[\mathrm{MPa}]$ & $E^{\prime}[\mathrm{MPa}]$ & $\sigma_{Y}[\mathrm{MPa}]$ & $v$ \\
& 210300 & 5123 & 273.3 & 0.3 \\
\hline
\end{tabular}

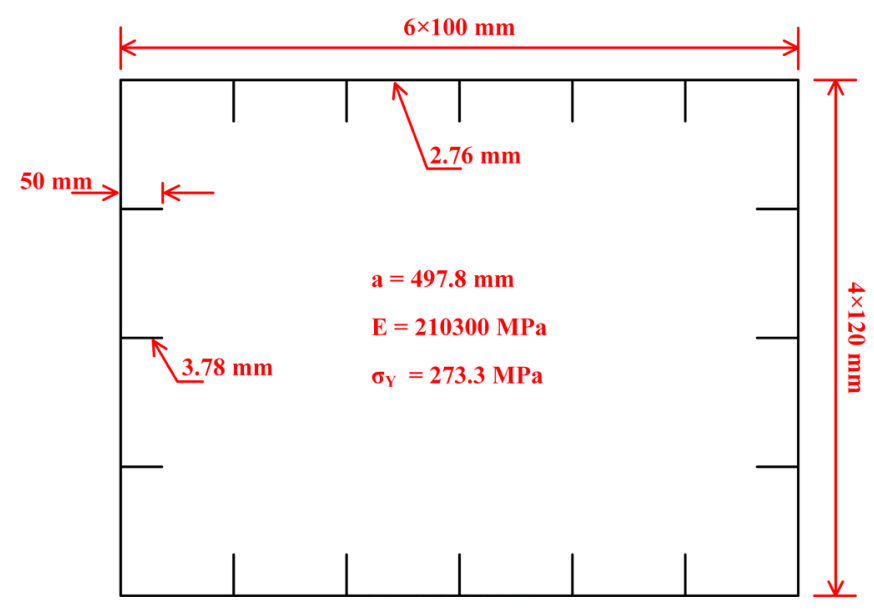

Figure 10. Cross section of the experimental model 


\subsection{Numerical Model Characteristics}

Four box girder models are adopted for the numerical validation. These box girders are simplified representations of conventional midship cross sections for a single hull, double hull, bulk carrier and container ship (Figure 11). With these simplified cross sections, the computational demand is considerably reduced while representative ship structural responses can be predicted. Each cross section has a width of $12000 \mathrm{~mm}$ and a height of $8000 \mathrm{~mm}$. The thicknesses of the outer and the inner plating are $12 \mathrm{~mm}$ and $10 \mathrm{~mm}$ respectively. Except for the stiffening of the longitudinal girders in the double bottom where flat-bar stiffeners are utilised, the cross section is longitudinally stiffened by teebar stiffeners with the same scantling. In addition, each model is transversely framed at a spacing of $1500 \mathrm{~mm}$. The scantlings and material properties of the numerical models are summarised in Table 3.

Table 3. Dimension and material property of the numerical model

\begin{tabular}{ccccccc}
\hline \multirow{5}{*}{ Geometry } & & $a(\mathrm{~mm})$ & $h_{w}(\mathrm{~mm})$ & $t_{w}(\mathrm{~mm})$ & $b_{f}(\mathrm{~mm})$ & $t_{f}(\mathrm{~mm})$ \\
& Tee-bar & 1500 & 150 & 8 & 70 & 15 \\
& Flat-bar & 1500 & 100 & 10 & $/$ & $/$ \\
& Trans. frame & 1500 & 300 & 16 & 140 & 30 \\
\hline \multirow{2}{*}{ Material } & $E[\mathrm{MPa}]$ & $\sigma_{Y}[\mathrm{MPa}]$ & $v$ & & & \\
& 207000 & 315 & 0.3 & & & \\
\hline
\end{tabular}

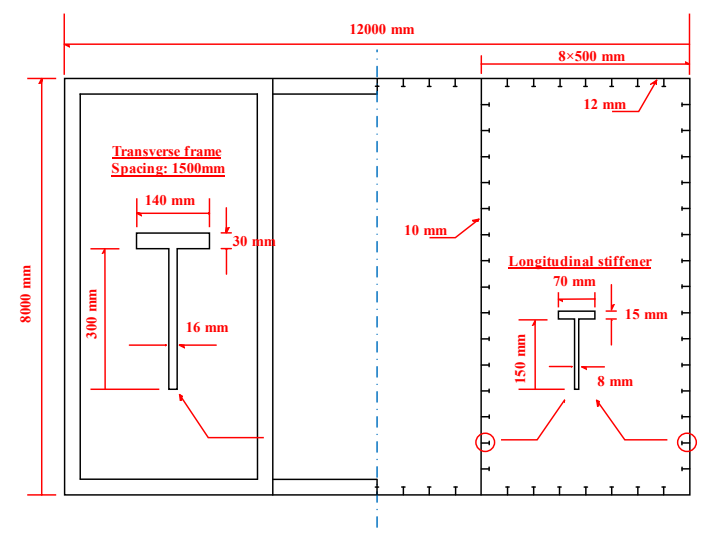

(a) Single Hull

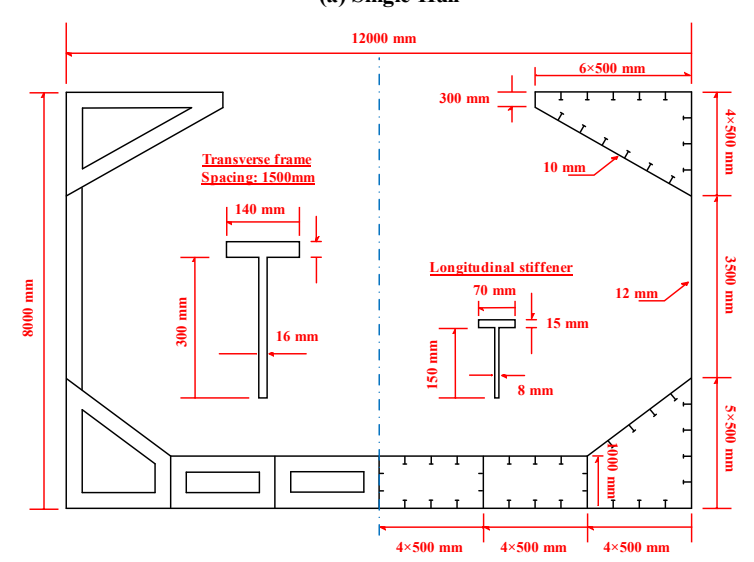

(c) Bulk Carrier

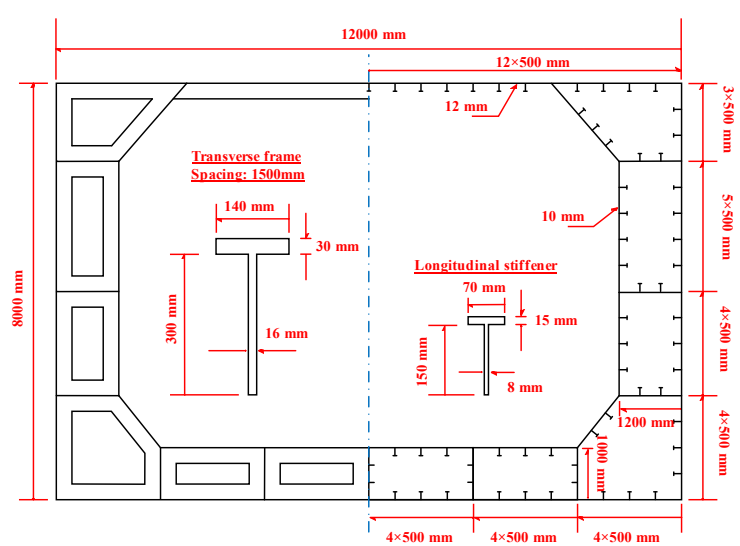

(b) Double Hull

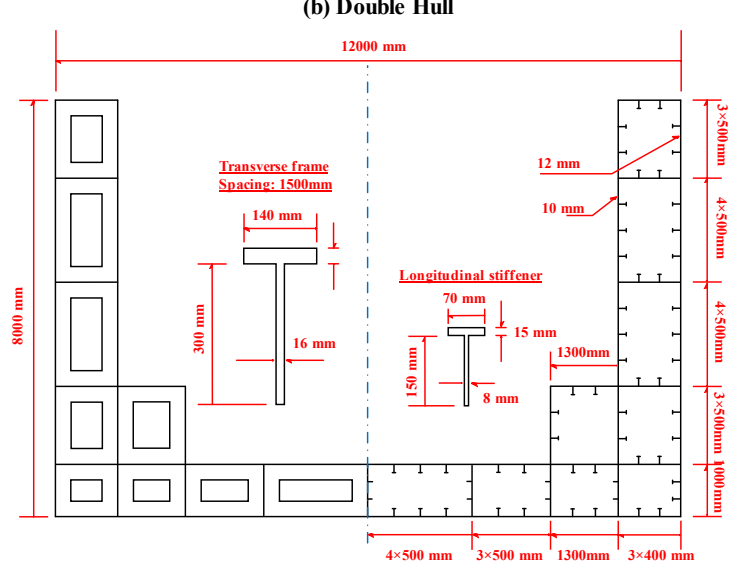

(d) Container Ship

Figure 11. Cross sections of the box girder models 


\subsection{Finite Element Modelling}

A three-bay model is adopted for the hull girder response numerical prediction. This model extent can include the effect of the transverse frame while minimising the overall model size and degrees of freedom, as discussed in [38]. For the plating discretisation, fifteen and five elements along the length and the breadth are utilised respectively. Two elements are used for the web and flange. This discretisation scheme results in a characteristic mesh size of $100 \mathrm{~mm} \times 100 \mathrm{~mm}$.

The building block approach developed in [34] is employed for the finite element modelling. Four-node shell elements with reduced integration are adopted. Three types of initial geometric imperfection are applied on the FE model using a direct node translation technique, namely the local plate deflections $w_{\text {opl }}$, the column-type deflections $w_{o c}$ and the stiffener sideway deflections $w_{o s}$ (Figure 12). Equation (11) to (18) gives the expressions of the deflection shapes and magnitudes. No welding-induced residual stress is considered.

To ensure buckling nucleates away from boundaries, the geometric imperfection is only applied on the middle bay of the hull girder model. For the application of load an arbitrary reference point is created, which ties to one end of the model as rigid body. There is no constraint applied on the reference point for loading application. The rotational displacement is applied on the reference point and the other end of the model is fixed by constraining six degrees of freedom. This technique automatically accounts for the shift of neutral axis in the way that the equilibrium of the resultant compression and tension is ensured.
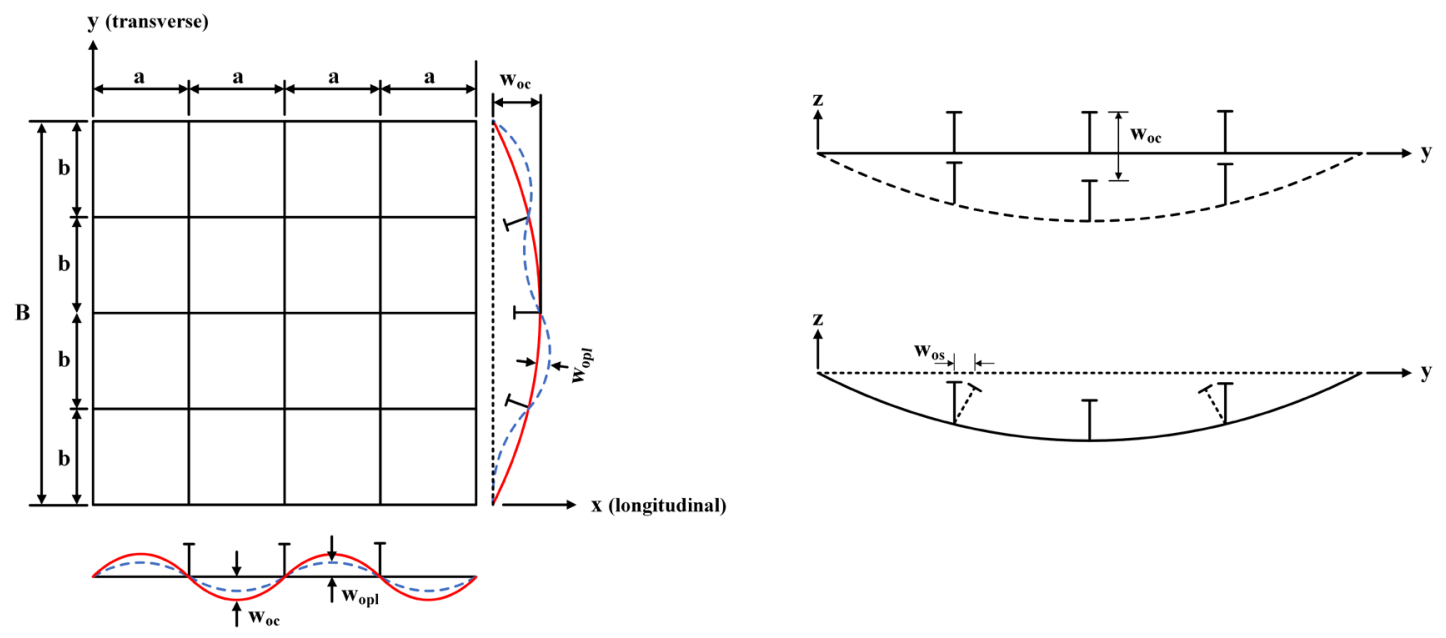

Figure 12. Schematic illustration of the initial geometric imperfections

$$
\begin{aligned}
& w_{o p l}=A_{o}\left\{0.8 \sin \left(\frac{\pi x}{a}\right)+0.2 \sin \left(\frac{m \pi x}{a}\right)+0.01 \sin \left[\frac{(m+1) \pi x}{a}\right]\right\} \sin \left(\frac{\pi y}{b}\right) \\
& m=\operatorname{int}\left(\frac{a}{b}\right)+1 \\
& A_{o}=\min \left(6,0.1 \beta^{2} t\right)
\end{aligned}
$$




$$
\begin{aligned}
& \beta=\frac{b}{t} \sqrt{\frac{\sigma_{Y}}{E}} \\
& w_{o c}=B_{o} \sin \left(\frac{\pi x}{a}\right) \sin \left(\frac{\pi y}{B}\right) \\
& B_{o}= \begin{cases}0.0008 a, & \lambda<0.2 \\
0.0012 a, & 0.2<\lambda<0.6 \\
0.0015 a, & \lambda \geq 0.6\end{cases} \\
& w_{o s}=C_{o} \frac{z}{h_{w}}\left[0.8 \sin \left(\frac{\pi x}{a}\right)+0.2 \sin \left(\frac{i \pi x}{a}\right)\right] \\
& i=\operatorname{int}\left(\frac{a}{h_{w}}\right)+1 \\
& C_{o}=0.002 a
\end{aligned}
$$

\subsection{Validation of Structural Component Load-Shortening Response}

As introduced in Section 3.2, the initial compressive load-shortening curve (LSC) of structural elements must be pre-defined as an input of the overall calculation methodology. Three different ways of predicting this initial LSC are used in the following analysis (Table 4). LSC 1 is generated using CSR method and LSC 2 is produced by static-Riks FEA. These two LSCs are more comparable as both are static analysis-based and assume elastic-perfectly plastic material behaviour. On the other hand, LSC 3 is calculated with the same FEA model of producing LSC 2, but through the dynamic implicit solver with consideration of Chaboche material hardening model (Table 1). It is used in one numerical test case to elucidate the effect of material hardening.

As the scantling of transverse frame is not available in [15], a single bay plate-stiffener combination is utilised for the nonlinear FEA of stiffened panel elements of experimental model. The single-bay model is discretised with 50 four-node shell elements along its length, 10 elements along its breadth and 5 elements along the height of stiffener's web, resulting in a $10 \mathrm{~mm} \times 10 \mathrm{~mm}$ characteristic element size. On the other hand, $a 1 / 2+1 / 2$ bay model with transverse frame is used for the stiffened panel elements of numerical models. The $1 / 2+1 / 2$ bay model is discretised with 60 four-node shell elements in along its length, 20 elements in its breadth and 6 elements along the height of stiffener's web, resulting in a $25 \mathrm{~mm} \times 25 \mathrm{~mm}$ characteristic element size. Boundary conditions of both models are illustrated in Figure 13. In the present NLFEM models, the pull-in of the long edges are restrained and the toe of the transverse frames are restricted on their transverse and vertical translations. The former effectively leads to a biaxial loading condition on the panel due to Poisson's effect, while the latter implies an infinitely rigid transverse support. These boundary conditions are liable to increase the initial stiffness and ultimate strength of the panels. Meanwhile, these may also induce an uncertainty in the capacity loss during post-collapse regime. As suggested by Smith et al. in [39], a biaxially loaded plate would exhibit a less drastic drop of the post-collapse strength. Hence, the adopted NLFEM models may have a relatively conservative estimation over the post-collapse response. 

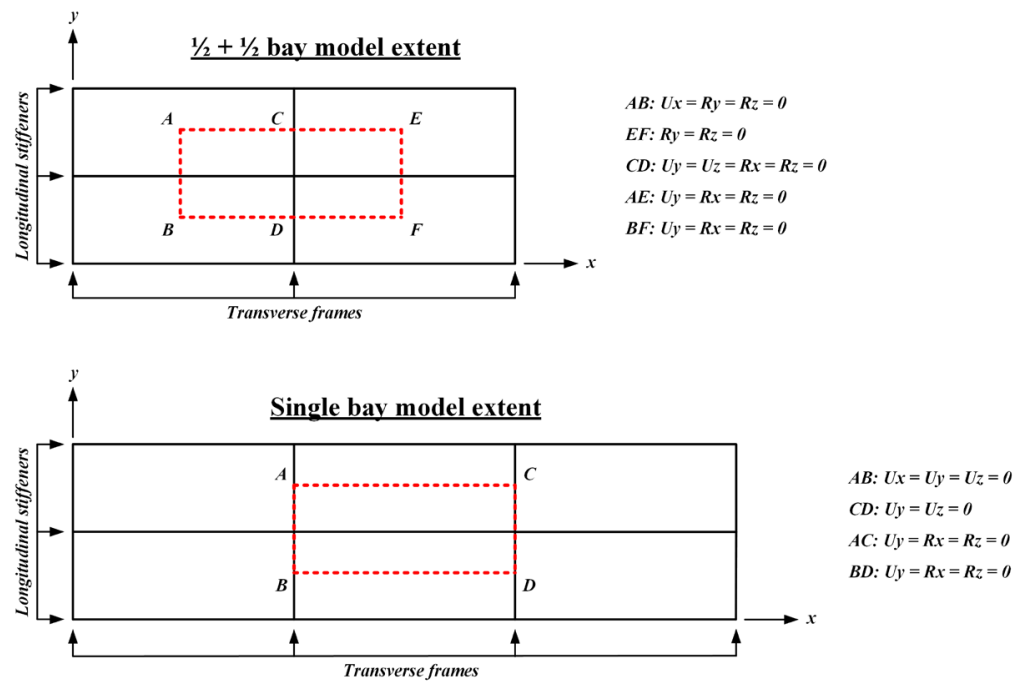

Figure 13. Model extent of plate-stiffener combination model

Table 4. Different LSCs used in the numerical validation

\begin{tabular}{ccc}
\hline No. & Methodology & Material behaviour \\
\hline LSC 1 & CSR method & Elastic-perfectly plastic \\
LSC 2 & Static-Riks FEA & Elastic-perfectly plastic \\
LSC 3 & Dynamic implicit FEA & Chaboche hardening \\
\hline
\end{tabular}

To validate the prediction of structural component load-shortening response under cyclic loading, a comparison of load-shortening curves of the deck/bottom panels under cyclic loading $\left(-1.8<\varepsilon / \varepsilon_{Y e q}<\right.$ 1.8) between the proposed method in [36] and the nonlinear FEA is given in Figure 14 and Figure 15 for experimental model and numerical model respectively.

In Figure 14 and Figure 15, the results predicted by the method developed in [36] is depicted by as "Proposed" with the bracket indicating the embedded initial compressive LSC. The nonlinear FEA herein refers to the dynamic implicit solver prediction with consideration of Chaboche hardening. A satisfactory agreement may be found in these comparison, as the principle collapse behaviours of a stiffened panel under extreme cyclic load are well captured. The in-plane stiffness of the unloading path closely correlates with the nonlinear FEA results. As a conventional practice, the tensile ultimate strength is assumed as the material yield stress which therefore leads to certain discrepancy with respect to the FEA estimation. Meanwhile, the comparison also highlights the uncertainty on the compressive reloading strength prediction due to the use of different initial LSC. As suggested by Figure 14(c) and 15 (c), the use of LSC 3 as an input results in a remarkably good correlation with nonlinear FEA, as both are based on dynamic implicit analysis considering material hardening. Despite LSC 1 and LSC 2 are more comparable in terms of their analysis assumptions (static-based and elastic-perfectly plastic), the former presents a better correlation of the reloading strength. This is primarily attributed to the difference in the post-collapse behaviour, as the reloading strength is taken as the same as the previous unloading stress from compression (Updating rule 7). A further validation on the compressive reloading strength is shown in Figure 16 for regular loading protocols with seven magnitudes $\left(-C<\varepsilon / \varepsilon_{Y e q}<C\right.$ with $C=1.2 \sim 1.8$ ). A reasonable correlation is obtained as indicated by the statistical analysis with a 
mean of 0.9621 and COV of 0.0812 for all test cases. Overall, the analytical method proposed in [39] could be concluded as a rational approach to simulate the collapse behaviour of structural component under extreme cyclic load and to provide input data for the hull girder progressive collapse analysis.

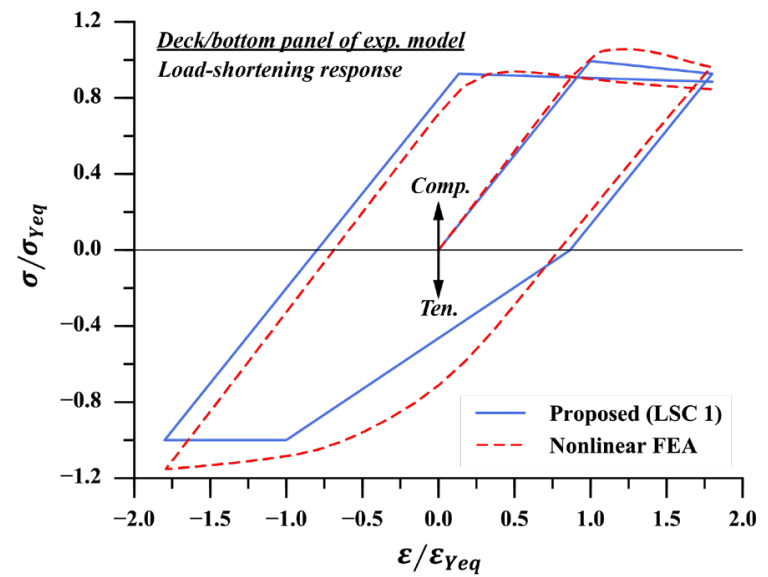

(a)

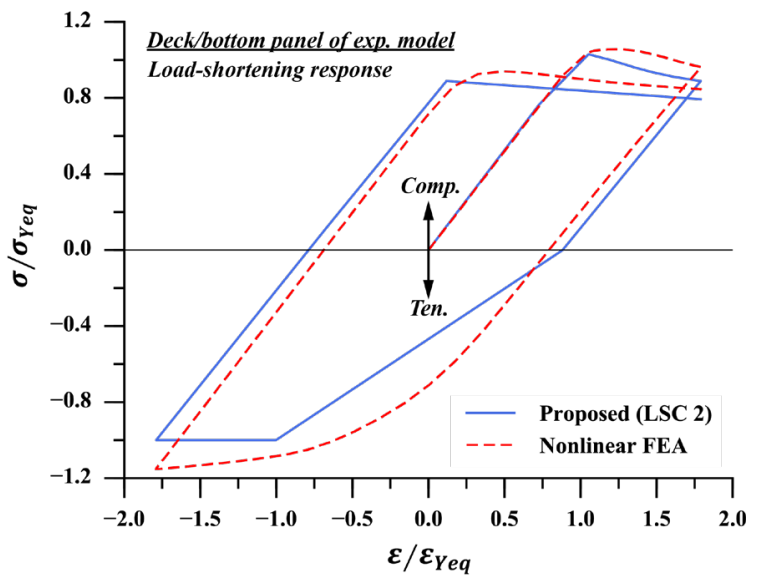

(b)

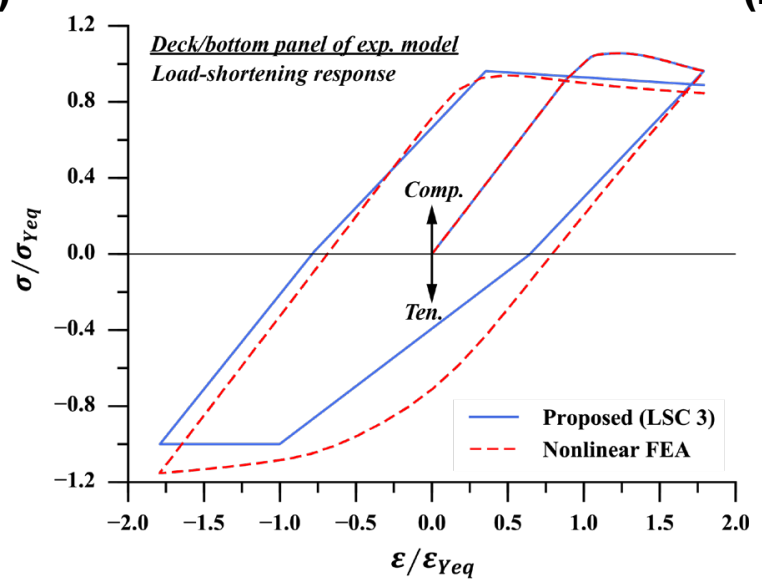

(c)

Figure 14. Load-shortening curve comparison of deck/bottom stiffened panel (Experiment model)

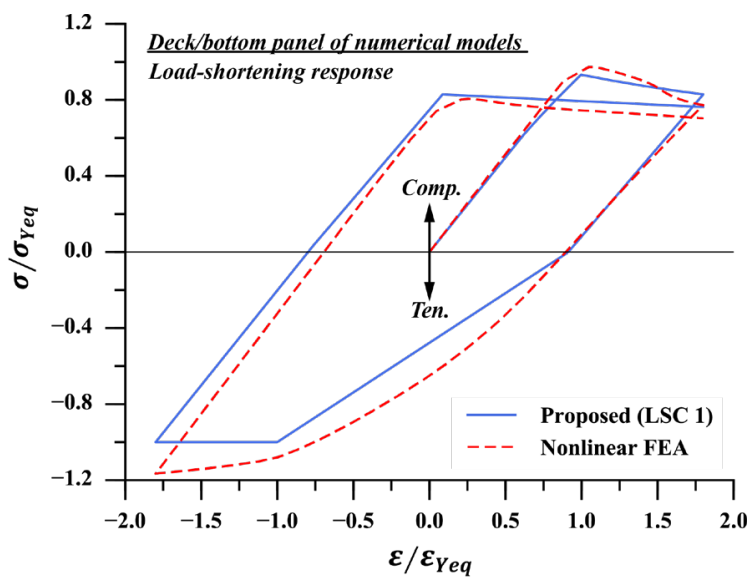

(a)

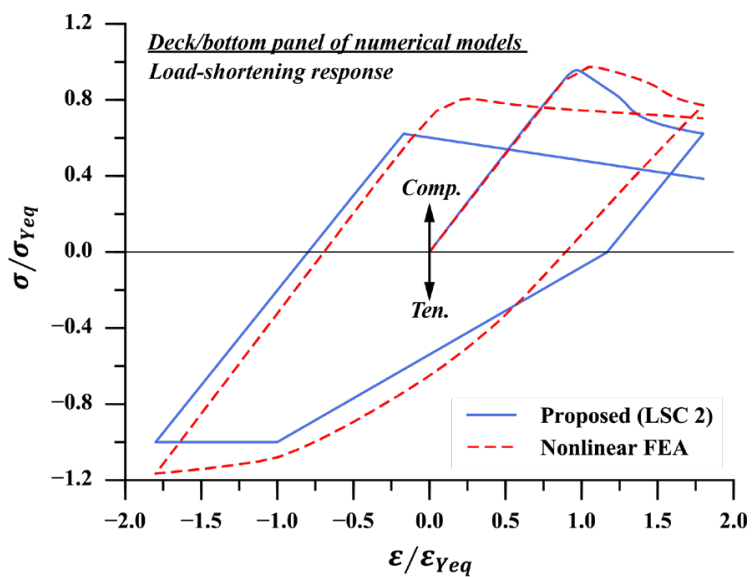

(b) 


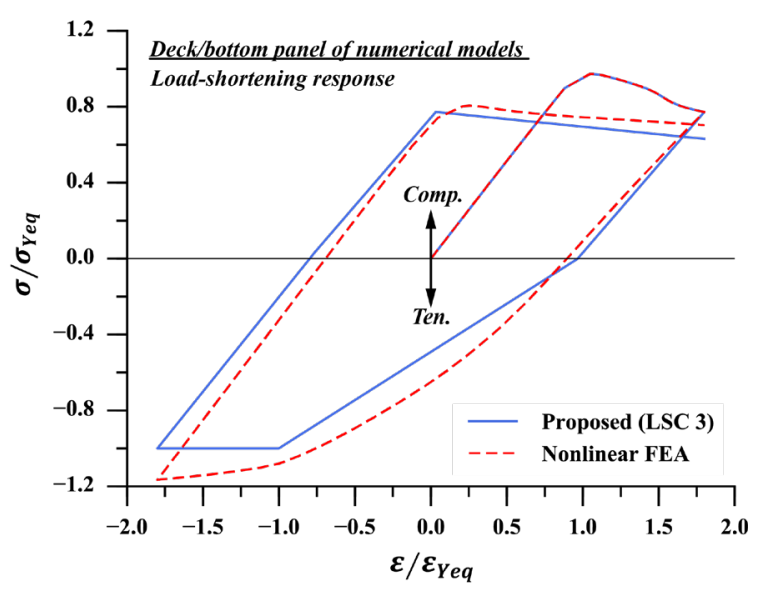

(c)

Figure 15. Load-shortening curve comparison of deck/bottom stiffened panel (Numerical model)

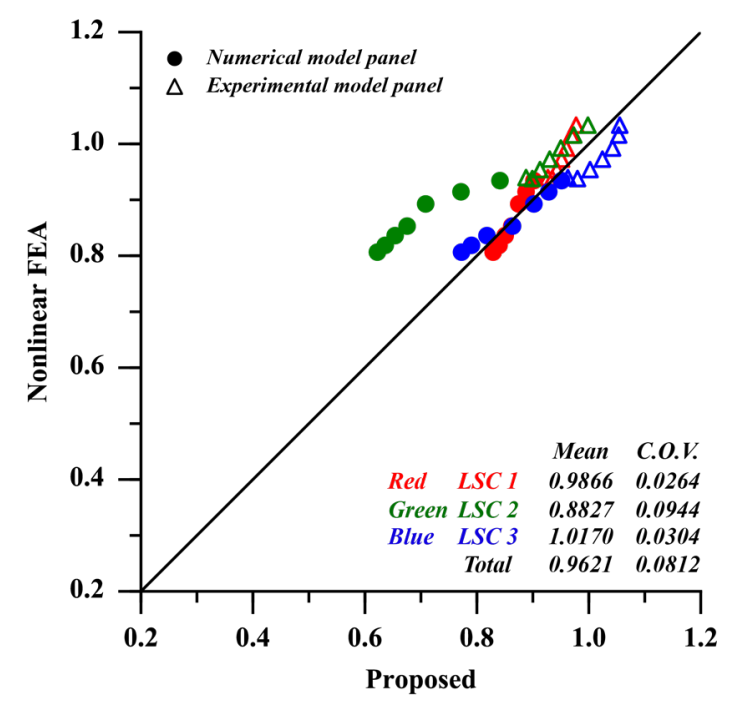

Figure 16. Correlation of the compressive reloading strength prediction

\subsection{Validation of Hull Girder Bending Response}

\subsubsection{Experimental Model}

The prediction of bending moment/curvature curves of experimental model are compared in Figure 17. In general, a reasonable agreement is obtained. A higher initial ultimate bending strength is predicted by the proposed cyclic progressive collapse method, which might be attributed to the effect of weldinginduced residual stress. In terms of the reloading to hogging at the first cycle, it appears that the reduction of ultimate bending strength is negligible, as indicated by the experimental measurement. However, a significant strength reduction is experienced in the second cycle. The sagging strength reduction of the second cycle is well correlated, whereas the prediction of hogging reloading strength in the second cycle by the cyclic progressive collapse method is relatively optimistic. The proposed method with LSC 1 gives a higher strength prediction in both cycles, which is primarily a result of the difference in the post-collapse response of load-shortening curve. The LSC 2 has a steeper post- 
collapse response, in comparison to the gentle capacity loss of LSC 1 as reflected in Section 4.4. The correlation of the bending strength between the predictions by the proposed method and nonlinear FEA is illustrated in Figure 18. Note that strength shown in Figure 18 are the max./min. values occurred at each loading cycle with the prescribed protocol. Owing to the effect of residual stress and uncertainty in initial distortion, the prediction by the proposed method is acceptably higher than the experimental measurement with an overall mean of 1.1468 and COV of 0.0841 .

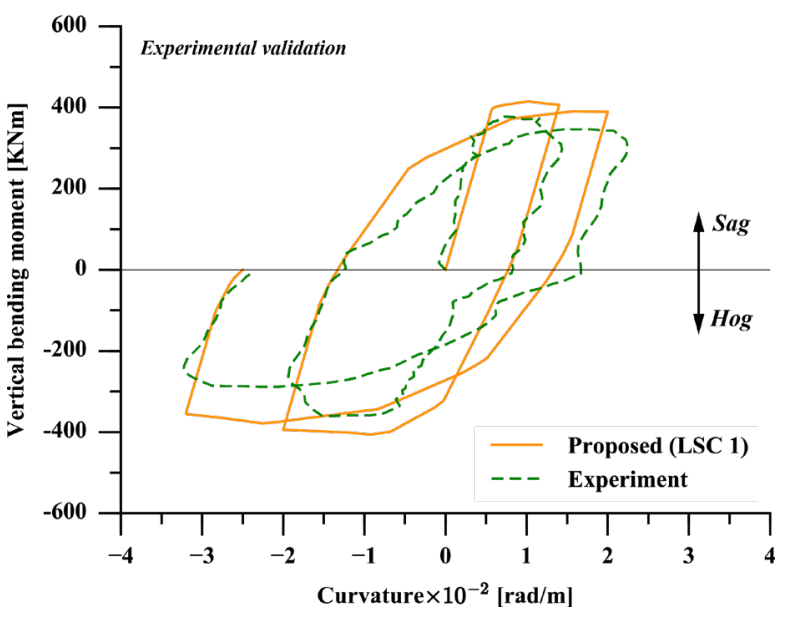

(a)

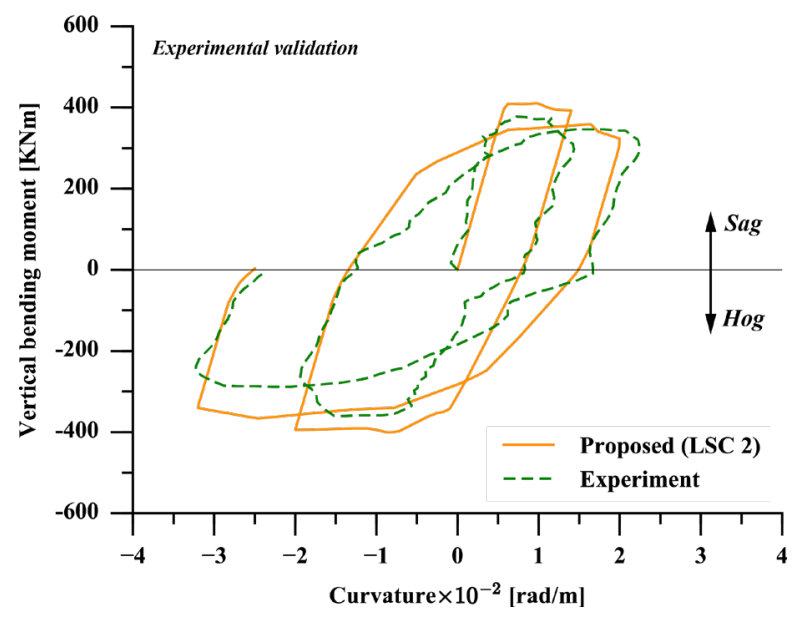

(b)

Figure 17. Comparison of bending moment/curvature relationships (Experimental model)

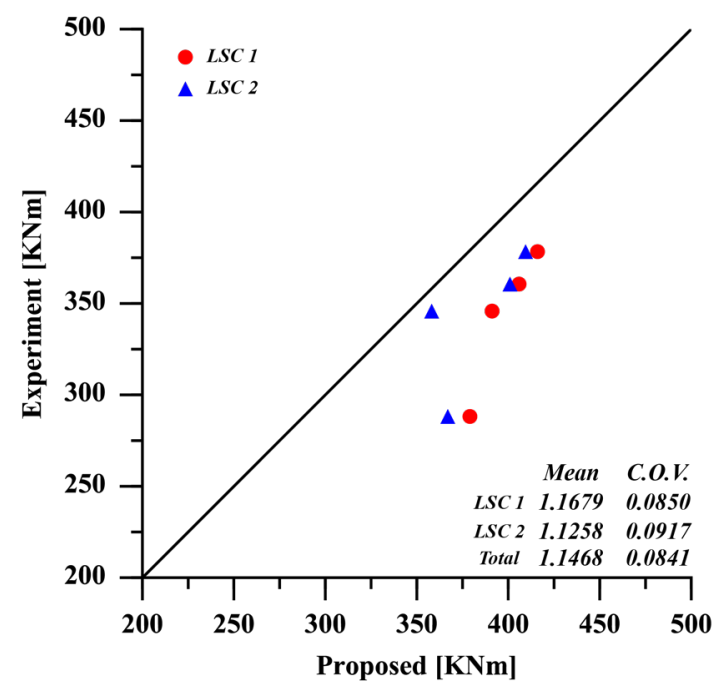

Figure 18. Correlation of the bending strength with experimental measurement (Experimental model)

\subsubsection{Numerical Model}

Two cyclic loading protocols are analysed for the numerical models. The applied curvature is started in sagging followed by an equal magnitude hogging load, after which a further reloading to sagging is carried out. The magnitudes of each loading protocol are $0.6 \mathrm{rad} / \mathrm{km}$ and $0.4 \mathrm{rad} / \mathrm{km}$ respectively. The former case corresponds to the condition where the ship hull girder is monotonically loaded far beyond 
the ultimate collapse state, whereas the latter case corresponds to the condition in which the ship hull girder is monotonically loaded close to the ultimate collapse state.

The hull FEA is completed with dynamic explicit solver at a simulation time of 500 seconds for each case to achieve quasi-static condition. Elastic-perfectly plastic material behaviour is assumed. The progressive collapse analysis using the proposed method is performed with the input of LSC 1 and 2 . In addition, to elucidate the cyclic hardening and Bauschinger effect, an extra nonlinear FEA with Chaboche hardening is carried out for single hull model and a comparison is made with the proposed method based on LSC 3. Figure 19 and 20 illustrates the typical deformation contour plots of the case study models at three limit states under two loading protocols where max./min. values occurred in the bending moment curves, namely the first-cycle sagging, first-cycle hogging and the second-cycle sagging. At the first-cycle sagging ultimate collapse state, the deck and top shell panels fail in a combination of beam-column and local plate buckling accompanied with plastic permanent set. The failure mode of the first-cycle hogging is similar to that under monotonic hogging, in which the deck panel experiences a gross yielding while the bottom panel is slightly buckled. For the second-cycle sagging ultimate collapse state, the buckling shapes of deck and top shell panels remain the same with an increase of the out-of-plane deflection. However, there is a change of the stress distribution in the top side shell panel when the hull girder is subjected to loading protocol 1. At the first-cycle ultimate sagging collapse state, a high stress level is experienced throughout the three-bay extent for both loading protocols. At the second-cycle, the high stress nucleates at the middle bay while the remaining part experiences a relatively low stress level when loading protocol 1 is applied.

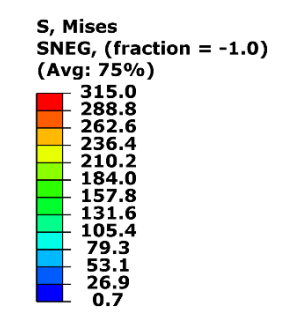

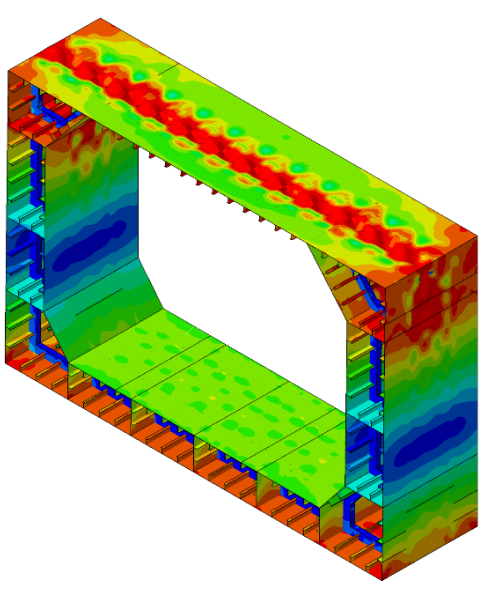

(a)

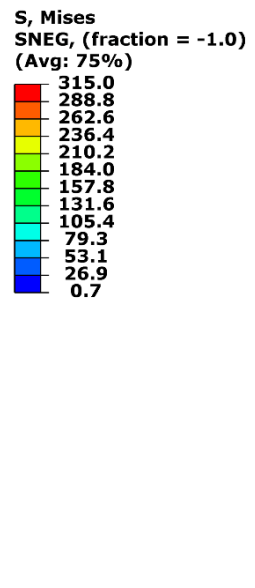

(b)

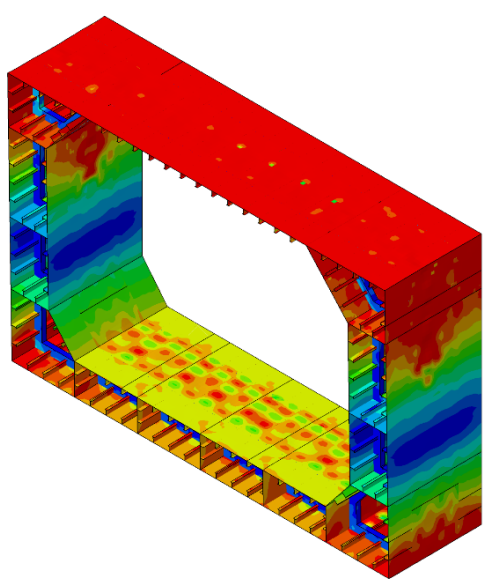




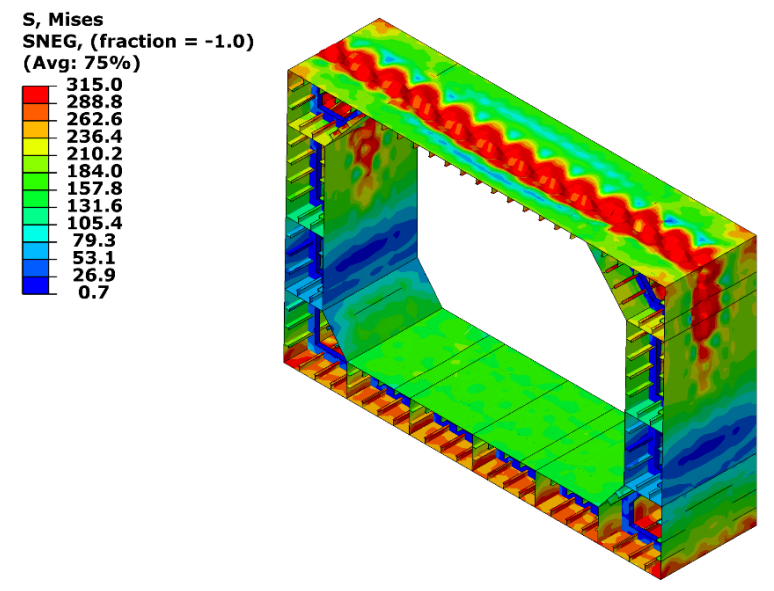

(c)

Figure 19. Contour plot under loading protocol 1
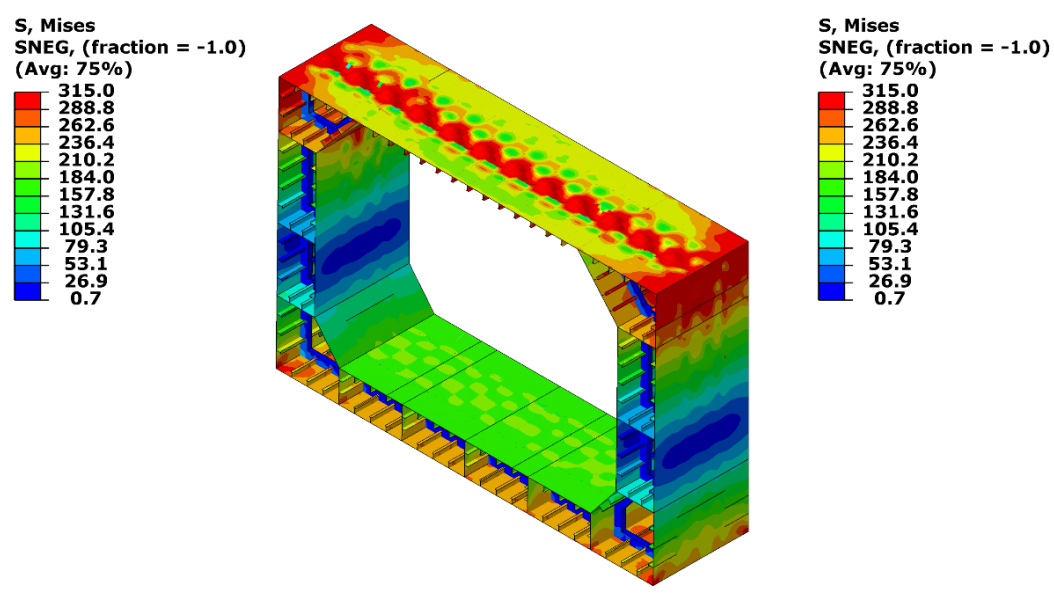

(a)
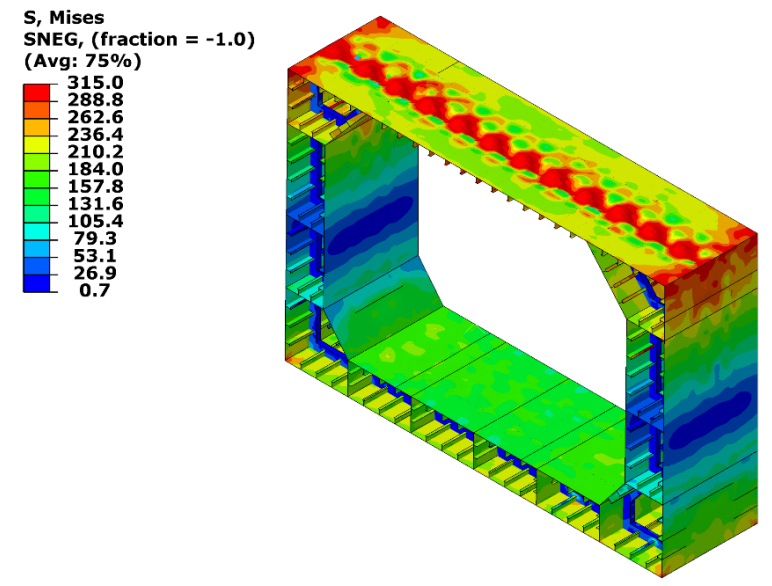

(b)

(c)

Figure 20. Contour plot under loading protocol 2

All the bending moment/curvature curve comparisons are shown in Figure 21 to Figure 24. For loading protocol 2, the cyclic progressive collapse analysis is only carried out using the LSC 2, since it is found 
that there is only an insignificant strength reduction when LSC 1 are used. When the cross section is initially loaded in sagging up to or beyond the ultimate limit state of sagging, there is a considerable strength reduction in the sagging reloading. On the other hand, the nonlinear FEA also predicts a reduction of the ultimate hogging strength. It seems that in general the cross sections with double side shell suffer from a larger strength reduction compared with the cross sections with single side shell. In terms of the reloading from sagging to hogging, it is clear that under loading protocol 1 the bending stiffness is lower than the monotonic hogging stiffness. As reviewed in Section 1, the bending stiffness of the reloading response was assumed to be the same as the initial stiffness in the hydro-elastoplastic approach developed in [10] because a circular solid bar was used as test specimen to model the nonlinear structural behaviour. However, the present analysis suggests that the structural stiffness of a thin-walled box girder would be reduced in the reloading phase because of the residual buckling deformation and residual stress.

In the progressive collapse analysis using the proposed method, the reduction of sagging strength is primarily attributed to the reduction of ultimate compressive strength of structural components during cyclic loading. When the LSC 2 is adopted, a better reloading sagging strength is predicted compared with the use of LSC 1 . However, during the reloading in hogging at the first loading cycle, the strength of each compressed components still remains as the same as the initial condition. Any variation of hogging strength is caused by the difference of the in-plane stiffness of the structural components under tensile loading, which leads to a difference of the neutral axis position and consequently a change on the hogging strength.

The effect of material hardening may be elucidated by Figure 21(c) which compares the bending moment/curvature curves predicted by nonlinear FEA incorporated Chaboche hardening and the proposed method based on LSC 3 . The results are closely correlated and it may suggest that the material hardening does not impose significant change on the overall bending response of a ship hull girder, but an expected increase of the bending strength.

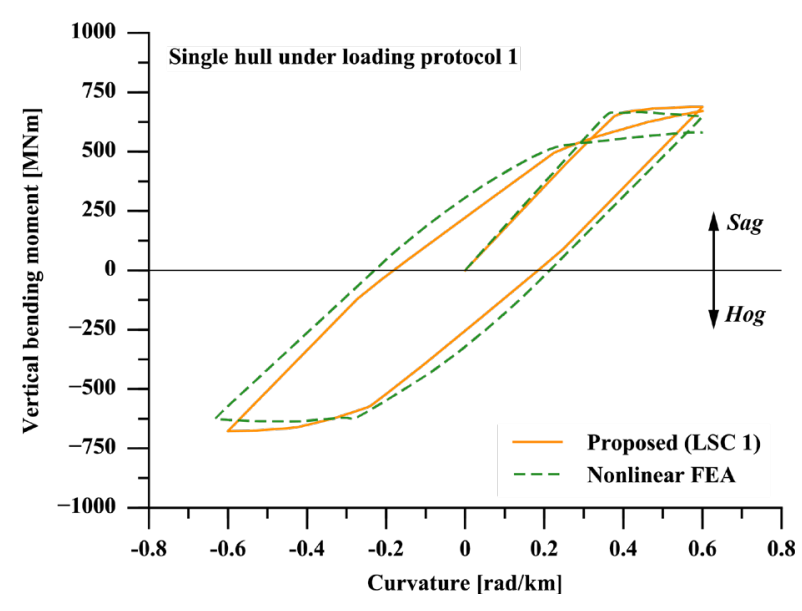

(a)

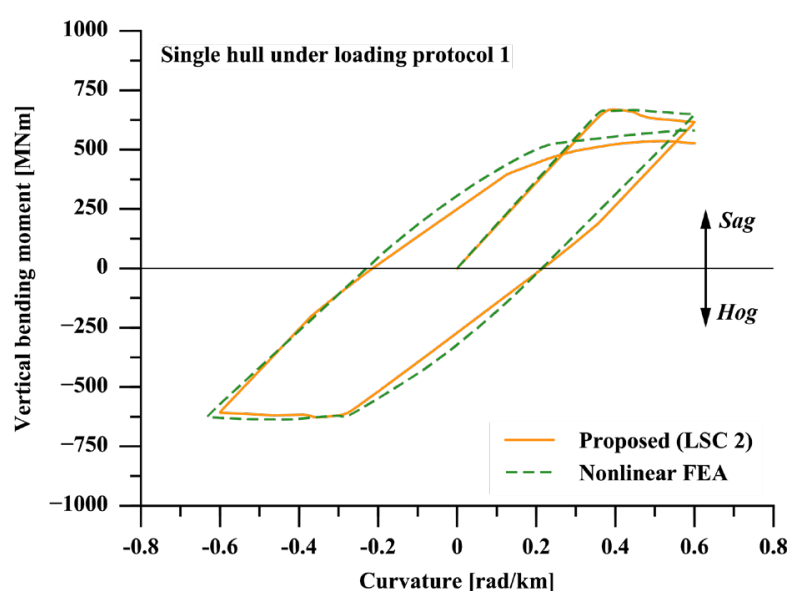

(b) 


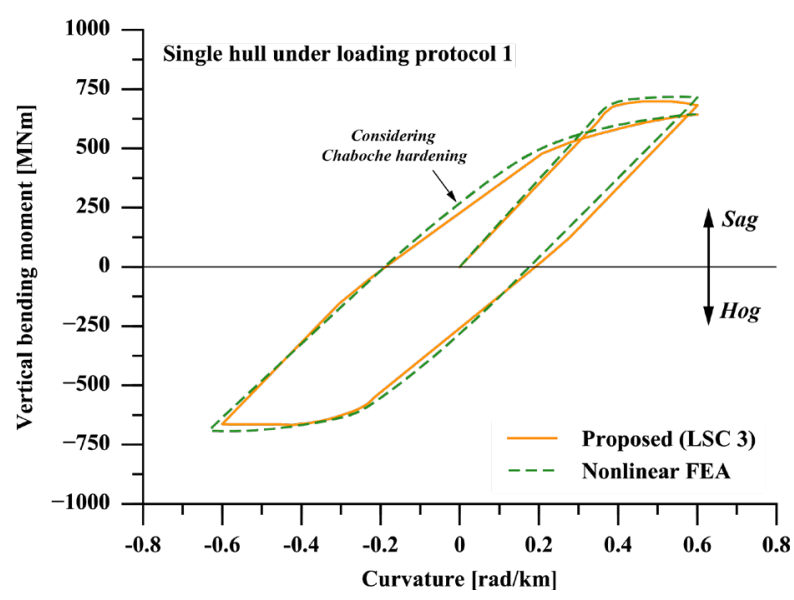

(c)

Figure 21. Single hull bending moment/curvature curves

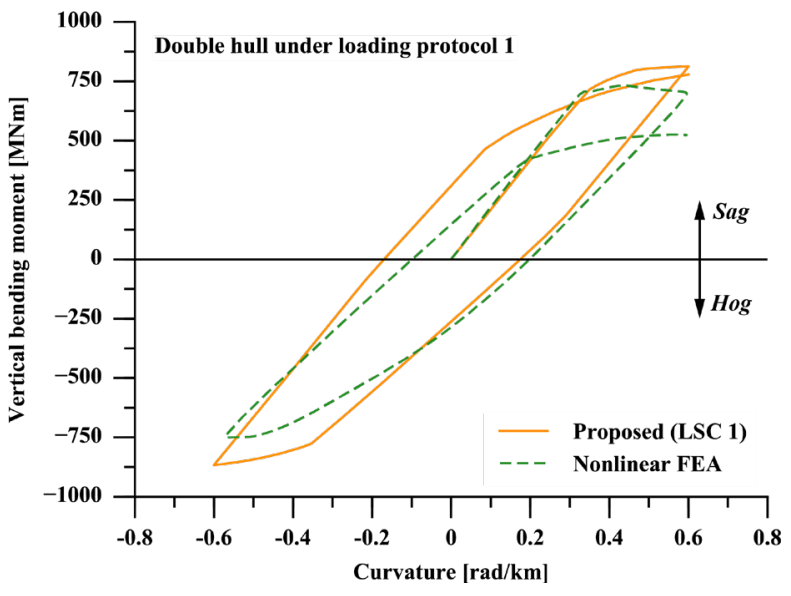

(a)

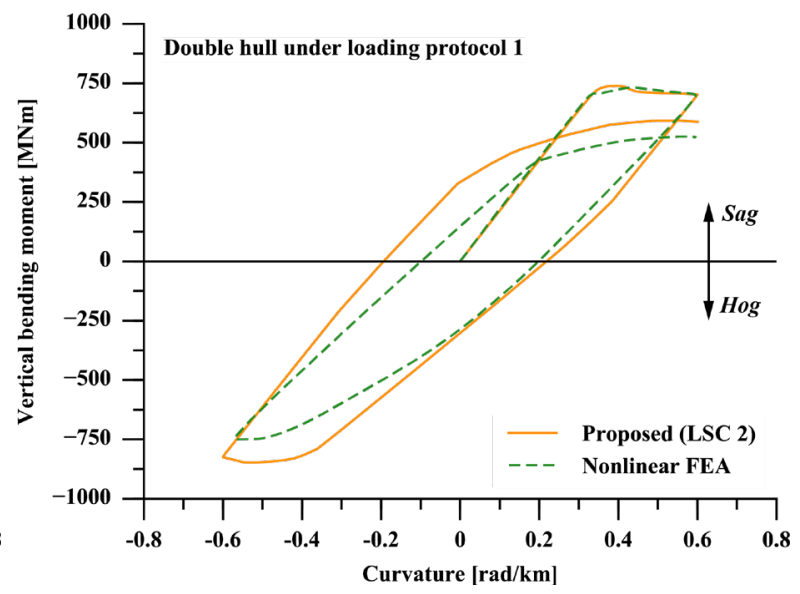

(b)

Figure 22. Double hull bending moment/curvature curves

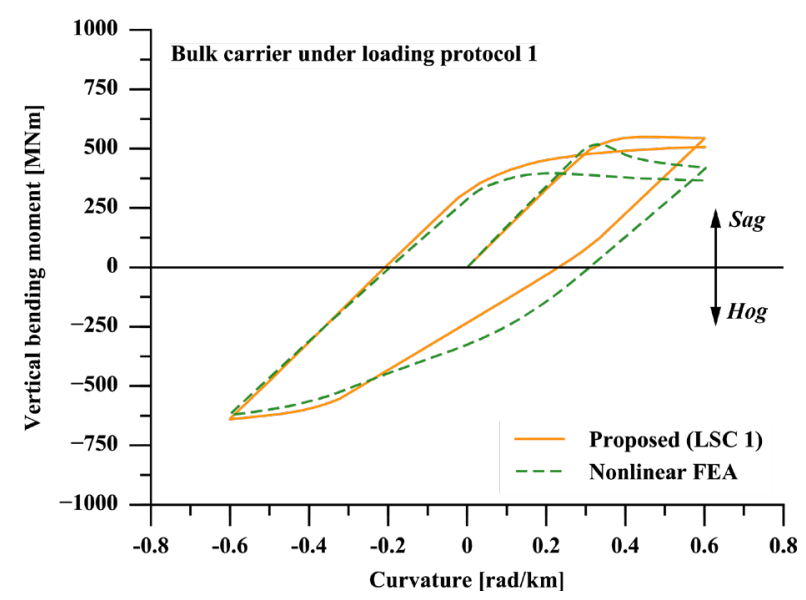

(a)

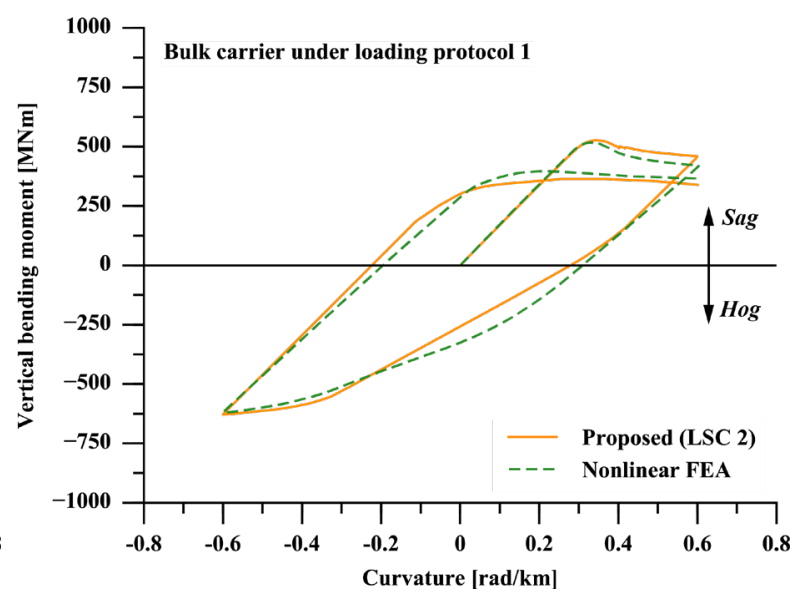

(b)

Figure 23. Bulk carrier bending moment/curvature curves 


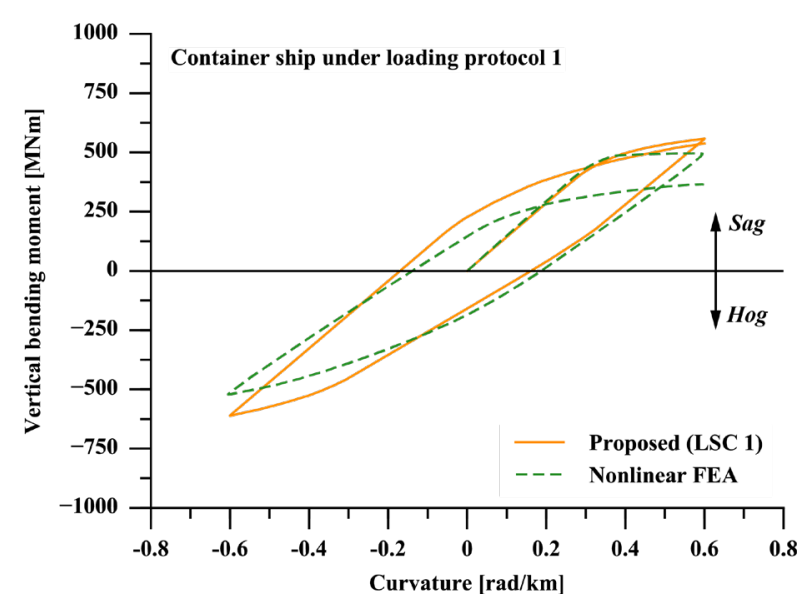

(a)

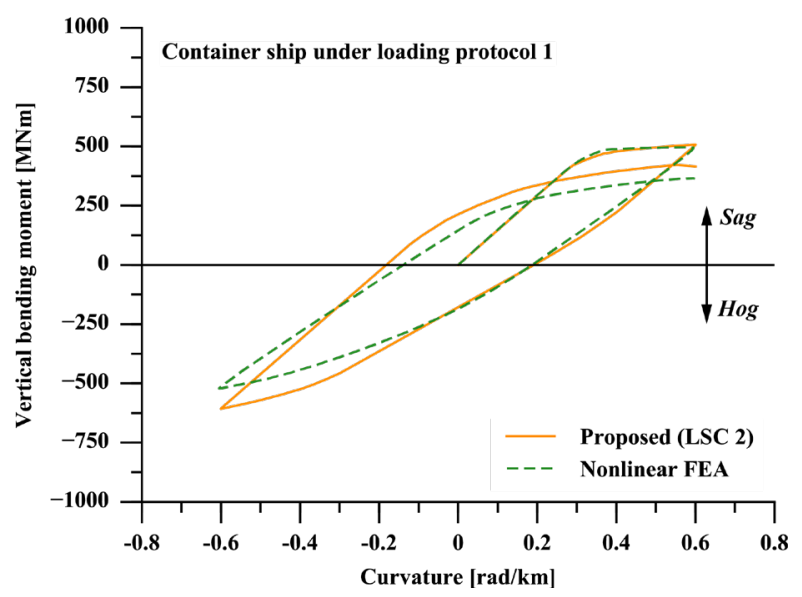

(b)

Figure 24. Container ship bending moment/curvature curves

As shown in Figure 25, the reloading strength predicted by the proposed method using the LSC 2 is reasonably correlated with the nonlinear FEA results giving a mean value of 1.1002 and COV of 0.1080 . Nevertheless, if LSC 1 is used, the predictions by the proposed method are optimistic with a mean value of 1.2252 and COV of 0.1396 . The discrepancy is also related to the difference of the post-collapse response of structural components, the effect of which has already been highlighted in the validation of structural component load-shortening response in Section 4.4. The statistical deviation is mainly associated with the results of double hull and container ship models, which have a double side shell cross section design. Apart from the fact that the present cyclic progressive collapse method might give a higher prediction on the hogging reloading strength, another source of discrepancy may be related to the uncertainty of nonlinear FEA. For example the loading protocol 2 corresponds to the condition where the hull girder is loaded close to the ultimate limit point, a significant difficulty might be encountered in the numerical analysis, which leads to a convergence issue.

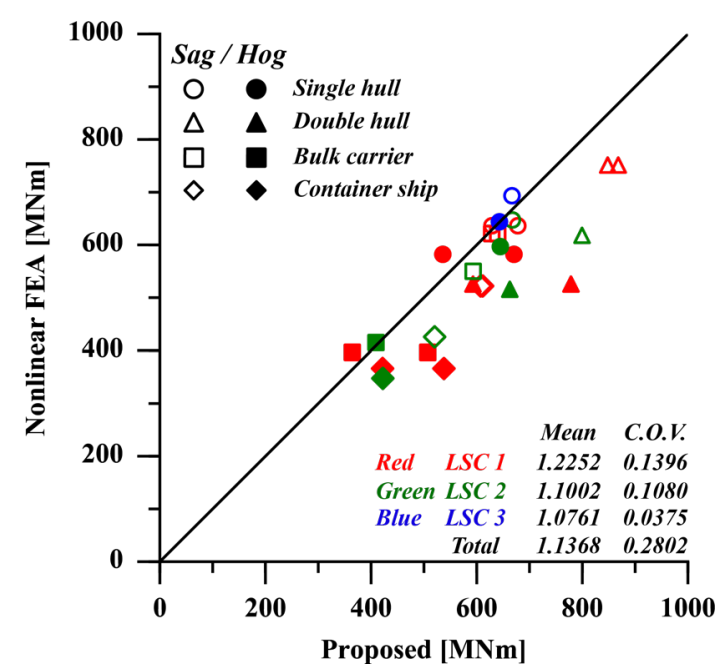

Figure 25. Correlation of the reloading bending moment with experimental measurement (Numerical models) 


\section{Ship Hull Girders under Multi-Cycle Loading}

In the previous section, a validation is completed through experimental measurement and numerical simulation, showing a relatively reasonable agreement on the prediction of single-cycle hull girder bending response. In this section, an attempt to investigate the hull girder response under multi-cycle loading using the proposed methodology is carried out. As shown in Figure 26, a comparison is first made on the variation of the compressive reloading strength of structural component under multi-cycle loading. The finite element modelling is consistent with Section 4.4. It can be seen that the convergence of compressive reloading strength is presented in all cases after three cycles of loading, whilst the magnitude of strength may differ due to the difference in post-collapse behaviour as highlighted previously. A further validation is given in Figure 27 comparing the multi-cycle bending moment/curvature curve predicted by the proposed method base on LSC 2 and nonlinear FEA. A relatively good agreement is shown in the general bending response path, which may give more confidence in the use of the proposed method to predict the multi-cycle ship hull bending response.

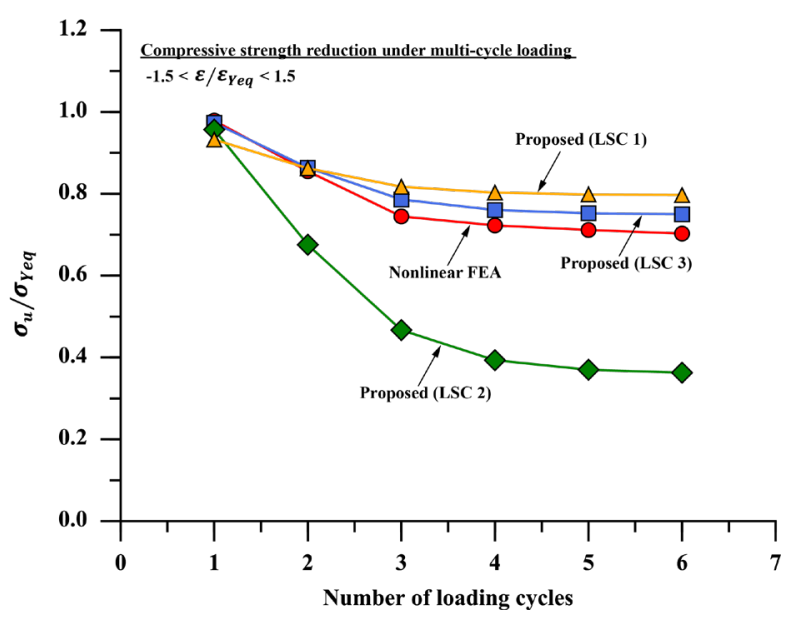

(a)

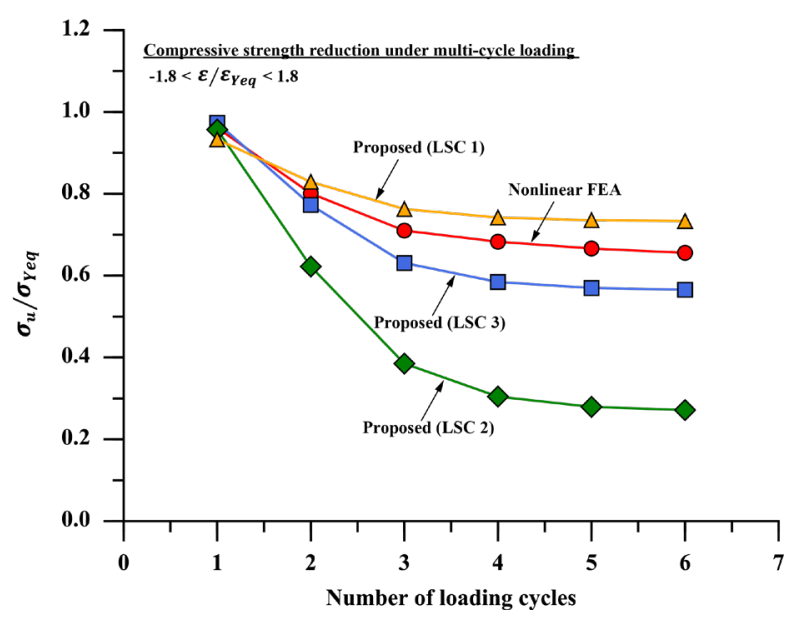

(b)

Figure 26. Compressive strength variation of structural component under multi-cycle compression and tension

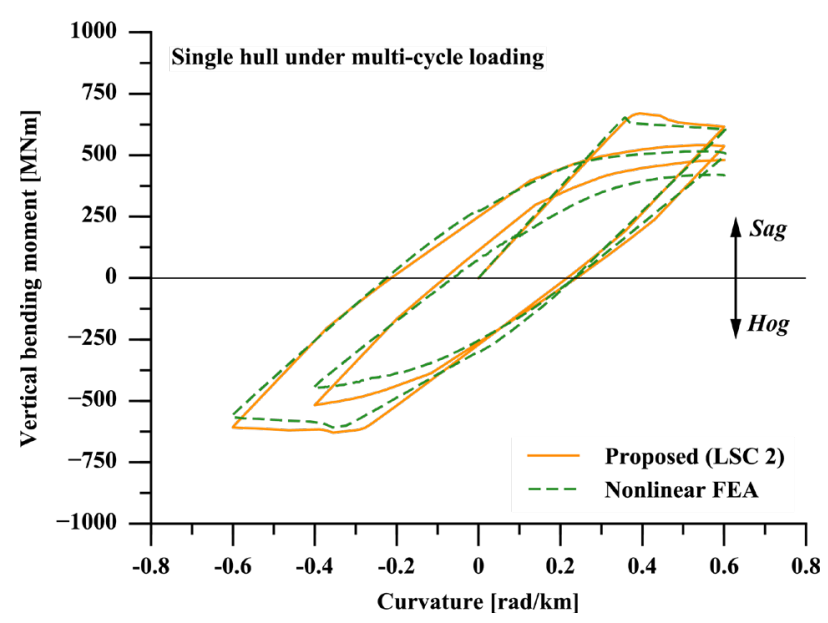

Figure 27. Comparison of bending moment/curvature curve of single hull girder under multi-cycle loading 
Typical multi-cycle bending moment curves of the numerical models predicted by the proposed method are shown in Figure 28. It is clear that the reduction of sagging strength is greater than hogging strength reduction. For bulk carrier-type and container ship-type models, the multi-cycle loading only induces a relatively minor hogging strength reduction. Figure 29 shows the variation of the maximum sagging strength as a function of the number of loading cycles. The degradation of sagging strength levels off after three cycles of loading. This behaviour agrees with the observation on structural components under cyclic in-plane load. Additionally, the numerical models with large deck opening appears to be relatively insensitive to different loading protocols, as the variation of the sagging strength are similar under both loading protocols.

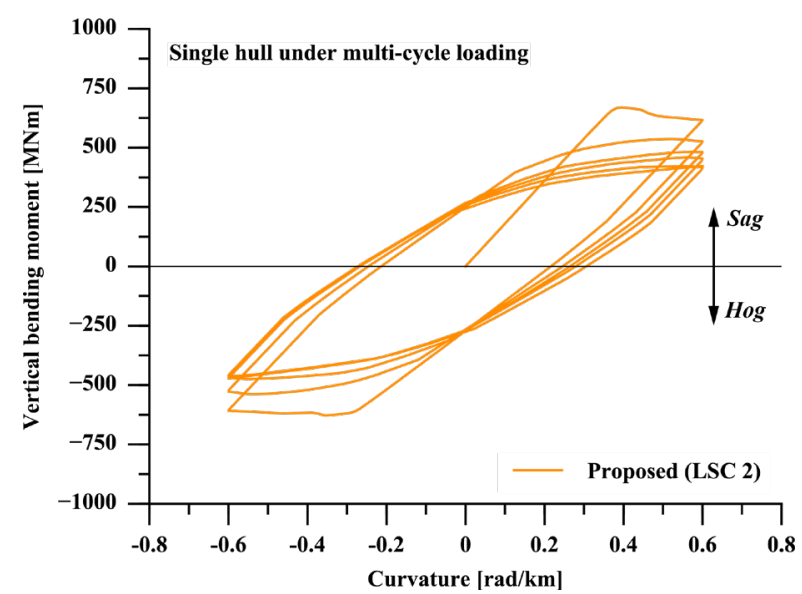

(a)

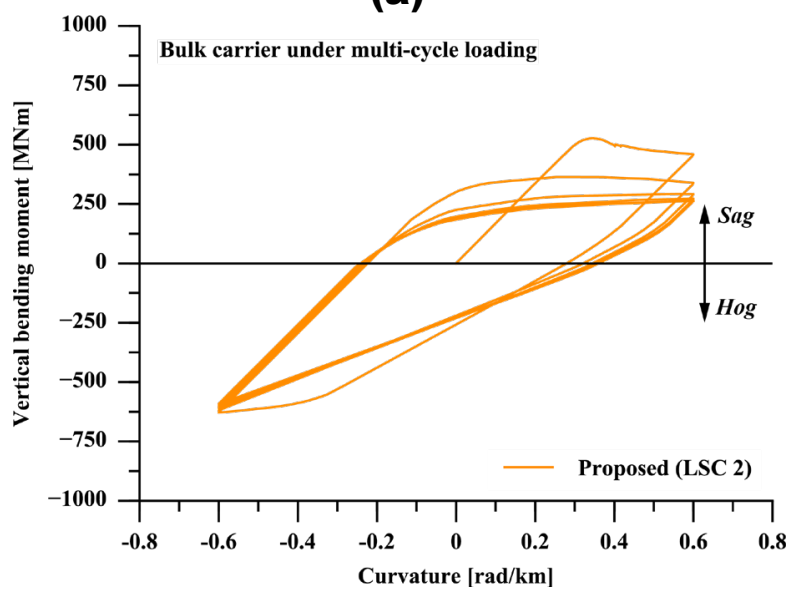

(c)

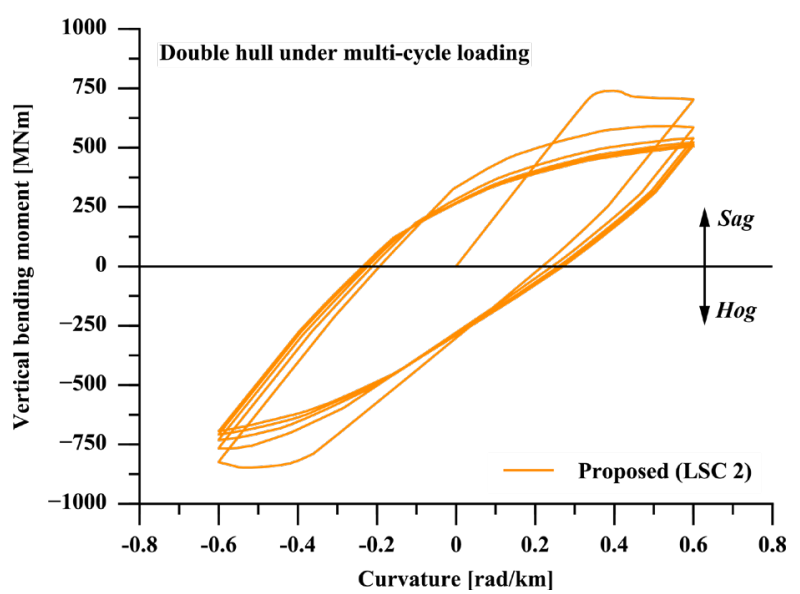

(b)

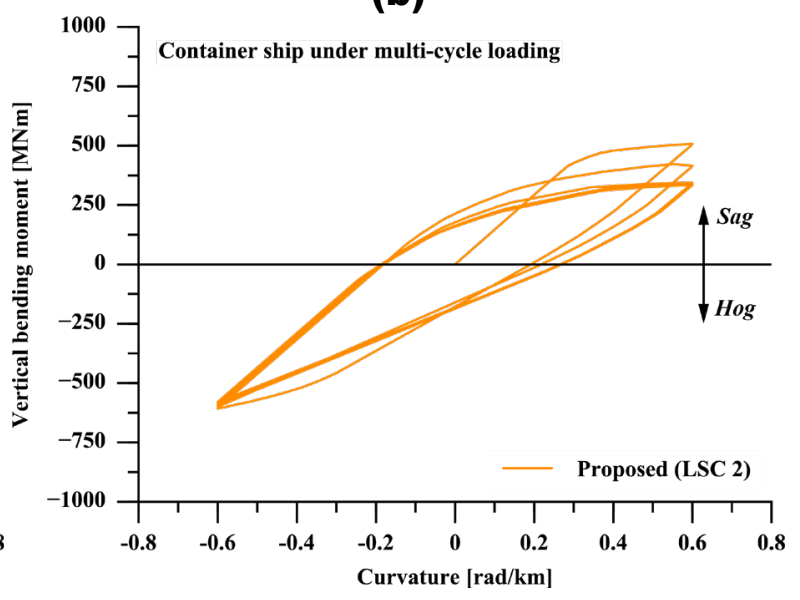

(d)

Figure 28. Typical bending moment/curve curves of hull girder under multi-cycle bending 

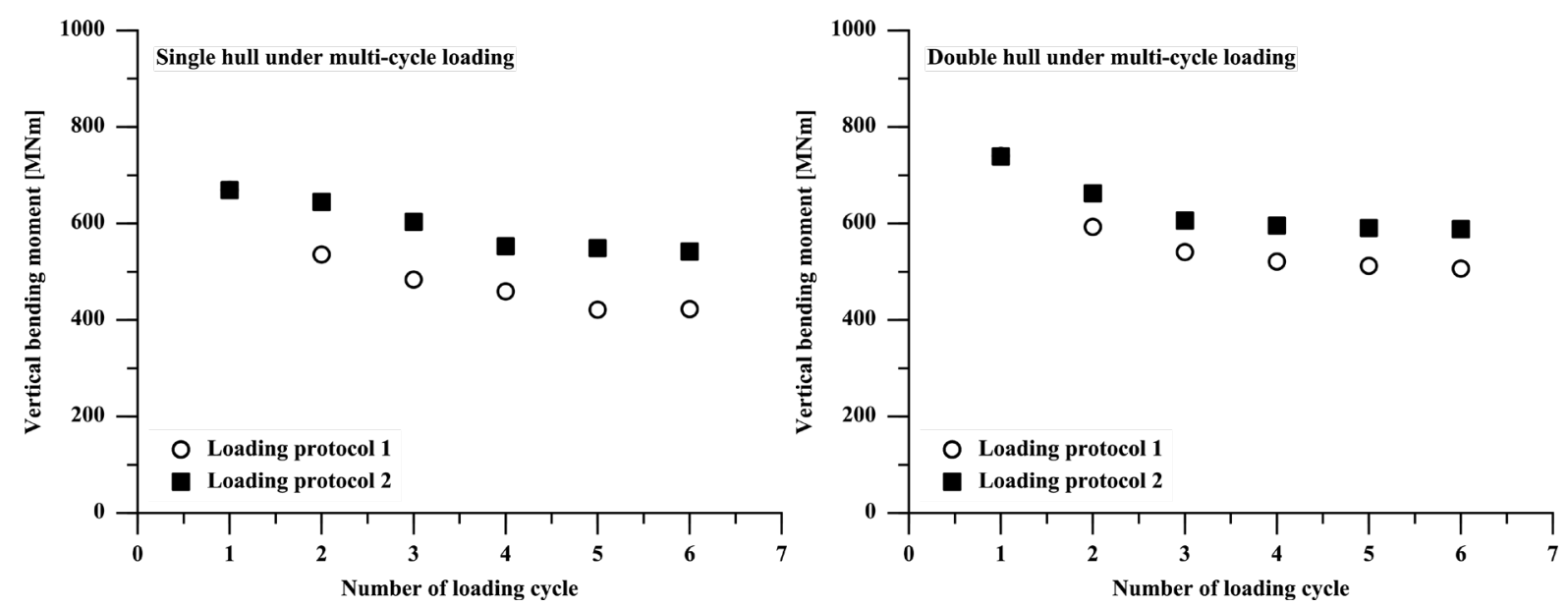

(a)
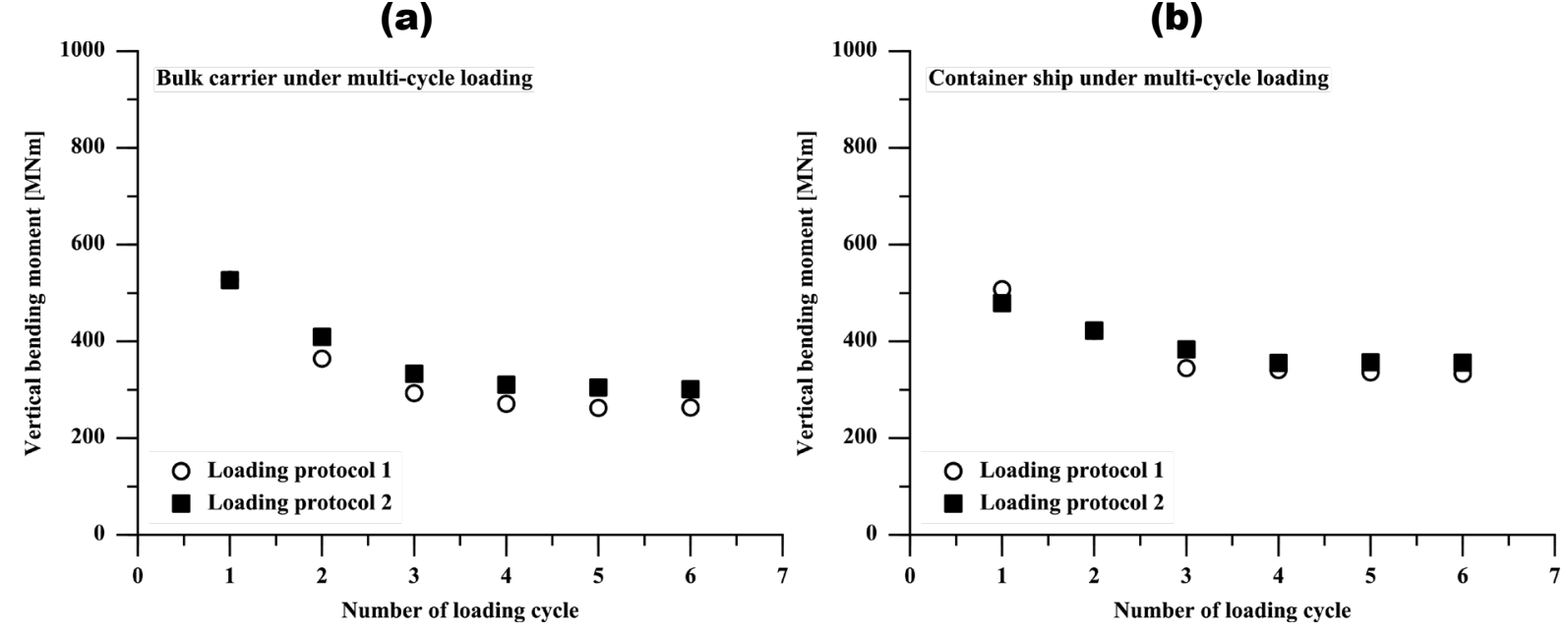

(c)

(d)

Figure 29. Variation of the sagging strength during the cyclic loading

\section{Conclusions}

This paper develops a novel cyclic progressive collapse method to predict the response of a ship hull girder under several cycles of extreme bending moment. The proposed method extends the capability of the Smith method through a response and updating reformulation rule for the load-shortening curves of structural components which is then coupled to the incremental-iterative algorithm of the progressive collapse method. Validations are completed using both experimental data and numerical simulations for comparison. The strength reduction under multi-cycle loading of several ship-type box girders is investigated. The following can be concluded from these case study analyses:

- The cyclic progressive collapse method provides a reasonable prediction of the cyclic bending response of a ship hull girder when compared to equivalent nonlinear FEA;

- When a FEA-based LSC is utilised within the cyclic progressive collapse method, a close correlation is obtained in comparison with nonlinear FEA of the hull girder.

- When a CSR-based LSC is utilised in the cyclic progressive collapse method, the cyclic bending response prediction tends to be optimistic for the case studies analysed here. The discrepancy is mainly related to the difference in the post-collapse response of structural components; 
- A series of cyclic loads which exceed the ultimate strength of the girder cause a significant reduction in the residual ultimate strength.

- The accumulative degradation of hull girder strength converges to a certain level after an initial reduction, which corresponds with the equivalent observation on the behaviour of structural components under cyclic in-plane load;

The analyses completed in this study are applied to simple representations of ship structures and are limited to a single extreme loading protocol which represents a more severe scenario than would be predicted by design guidelines such as the CSR. Nevertheless, the validation analyses indicate that the cyclic progressive collapse method is appropriate as a framework to assess the post-ultimate strength behaviour following a complex series of extreme load events.

Whilst the proposed cyclic progressive collapse method is reasonably validated in the present case study, it is necessary to further investigate the uncertainty caused by the different input of initial LSC, particularly the post-collapse response. According to the response and updating rule within the proposed method, the compressive reloading behaviour of structural components is highly affected by the post-collapse response of the previous cycle. A significant uncertainty may therefore be propagated into the prediction of hull girder reloading response. For instance, as shown in Section 4.4, the NLFEM models of stiffened panel elements are imposed with a rather conservative boundary condition in respect to their post-collapse response, which may then lead to an underestimation of hull girder strength. The influence of alternative LSCs based on different boundary conditions needs further investigation. Future work should also be conducted to investigate the effects of initial distortion and residual stress on the behaviour of structural components under extreme cyclic loading.

The extreme loading protocol used in this study is unrealistically severe for representing normal loading conditions on a ship designed to classification society rules. Whilst a strength degradation of the shiptype box girders is shown in the present study, it is necessary to complete further work to investigate if this type of cyclic loading history is relevant to the destructive collapse of a ship hull under extreme wave loading conditions, especially if these are substantially higher than the load scenarios embedded in contemporary classification rules and design philosophies.

In addition, it is of importance to consider the failure caused by ultra-low cycle fatigue in future work. An efficient criterion of ultra-low cycle fatigue may be developed and incorporated into the cyclic progressive collapse method.

The proposed cyclic progressive collapse method can provide an evaluation of the static bending moment/curvature relationship under extreme cyclic load. To consider the nonlinear response under a realistic loading protocol, this methodology could be coupled with a load protocol prediction method. Through this integration, the nonlinear response of a ship hull girder in an extreme sea state may be simulated. 


\section{References}

[1] International Association of Classification Societies (IACS), 2019. Common Structural Rules for Bulk Carriers and Oil Tankers.

[2] International Association of Classification Societies (IACS), 2015. Longitudinal Strength Standard for Container Ships.

[3] Smith, C.S., 1977. Influence of local compressive failure on ultimate longitudinal strength of a ship's hull. In: International Symposium on Practical Design of Ships and others Floating Structures (PRADS), Tokyo, Japan.

[4] Dow, R.S., 1991. Testing and analysis of a 1/3 scale frigate model. In: Advances in Marine Structures 2. Dunfermline, Scotland: Elsevier, $749-773$.

[5] 14th ISSC, 2000. Ultimate Strength. ISSC Committee III.1. Nagasaki, Japan.

[6] 15th ISSC, 2003. Ultimate Strength. ISSC Committee III.1. San Diego, United States.

[7] Hess, P.E., Adamchak, J.C., Falls, J., 1997. Failure analysis of an inland waterway oil bunker tanker. Survivability, structures and material directorate technical report. Naval sur-face warfare centre, Carderock division.

[8] Sumi, Y., Fujikubo, M., Fujita, H., Kawagoe, Y., Kidogawa, M., Kobayashi, K., Nakano, T., Iwano, J., Takahira, T., Tamura, K., Ueda, N., 2015. Final Report of Committee on Large Container Ship Safety, Japan.

[9] lijima, K., Fujikubo, M., 2015. Cumulative collapse of a ship hull girder under a series of extreme wave loads. Journal of Marine Science and Technology, 20 (3): $530-541$

[10] lijima, K., Kimura, K., Xu, W., Fujikubo, M., 2011. Hydroelastoplasticity approach to predicting the post-ultimate strength behaviour of a ship's hull girder in waves. Journal of Marine Science Technology, 16(4): $379-389$.

[11] Yao, T., Nikolov, P., I., 1990. Buckling/plastic collapse of plates under cyclic loading. Journal of the Society of Naval Architects of Japan, 168.

[12] Yao, T., Fujikubo, M., Nie, C., Kamiyama, S., 1995. Development and application of simple plate model to simulate collapse behaviour under thrust. Journal of the Society of Naval Architects of Japan, 178, 439-449 (in Japanese).

[13] Fukumoto, Y., Kusama, H., 1985. Cyclic bending tests of thin-walled box beams. In Proceedings of JSCE, Structural engineering/Earthquake engineering, 2.

[14] Kaminski, M., L., 1992. Cyclic compression of imperfect plates. PhD thesis, Delft University of Technology. 
[15] Cui, H., Yang, P., 2018. Ultimate strength and failure characteristics research on steel box girders under cyclic-bending moments. Journal of Marine Science and Technology.

[16] Caldwell, J.B., 1965. Ultimate longitudinal strength. Trans., RINA, 107, 411 - 430.

[17] Adamchak, J., 1982. ULTSTR: A program for estimating the collapse moment of a ship's hull under longitudinal bending. DTNSRDC report 82/076.

[18] Rutherford, S.E., Caldwell, J.B., 1990. Ultimate strength of ships: a case study. SNAME Trans., 98, $441-471$.

[19] Yao, T., Nikolov, P., 1991. Progressive collapse analysis of a ship's hull under longitudinal bending. Journal of the Society of Naval Architects of Japan, 172, $437-471$.

[20] Gordo, J.M., Guedes Soares, C., 1996. Approximate method to evaluate the hull girder collapse strength. Marine Structures, 9, $449-470$.

[21] Smith, M.J., 2008. Ultimate strength assessment of naval and commercial ships. DRDC Atlantic TM 2008 - 059, Defence R\&D Canada, Atlantic.

[22] Smith, C.S., Dow, R.S., 1986. Ultimate strength of a ship's hull under biaxial bending. ARE TR 86204, Admiralty Research Establishment, Dunfermline, Scotland.

[23] Benson, S., Downes, J., Dow, R.S., 2013. Compartment level progressive collapse analysis of lightweight ship structures. Marine Structures 31, $44-62$.

[24] Syrigou, M., Benson, S.D., Dow, R.S., 2018. Progressive collapse assessment of intact box girders under combined bending and torsional loads. In: International Conference on Ships and Offshore Structures, Gothenburg, Sweden.

[25] Fujikubo, M., Zubair Muis Alie, M., Takemura, K., lijima, K., Oka, S., 2012. Residual hull girder strength of asymmetrically damaged ships. Journal of Japan Society of Naval Architects and Ocean Engineers, 16, $131-140$.

[26] Dow, R.S., 1997. Structural redundancy and damage tolerance in relation to ultimate ship hull strength. In: Advances in Marine Structures 3, DERA, Dunfermline, Scotland.

[27] Dow, R.S., Smith, C.S., 1986. FABSTRAN: A computer program for frame and beam static and transient response analysis (Nonlinear), ARE report TR86205.

[28] Gordo, J.M., Guedes Soares, C., 1993. Approximate load shortening curves for stiffened plates under uniaxial compression. Integrity of Offshore Structures (Faulkner et al, Eds.), UK.

[29] Smith, M.J., 2010. A load shortening curve library for longitudinally stiffened panels. Technical Memorandum, DRDC Atlantic. 
[30] Ueda, Y., Rashed, S.M.H., 1984. The idealized structural unit method and its application to deep girder structures. Computers \& Structures, 18, $277-293$.

[31] Yao, T., Fujikubo, M., 2016. Buckling and ultimate strength of ship and ship-like floating structures. Elsevier.

[32] Chen, Y., Kutt, L., Piaszczyk, C., Bienek, M., 1983. Ultimate strength of ship structures. SNAME Trans., 91, $149-168$.

[33] Amlashi, J., Moan, T., 2008. Ultimate strength analysis of a bulk carrier hull girder under alternate hold loading condition - A case study Part 1: Nonlinear finite element modelling and ultimate hull girder capacity. Marine Structures, 21, $327-352$.

[34] Benson, S., Collette, M.D., 2017. Finite element methods and approaches. In: Carlton, J., Jukes, P., Choo, Y.S. (Eds.), Encyclopedia of Maritime and Offshore Engineering. John Wiley \& Sons, Ltd, Chichester, UK, $1-15$.

[35] Benson S, Downes J, Dow RS. An automated finite element methodology for hull girder progressive collapse analysis. In: 11th International Marine Design Conference (IMDC), 2012; Glasgow, UK.

[36] Li, S., Hu, Z., Benson, S., 2019. An analytical method to predict the buckling and collapse behaviour of plates and stiffened panels under cyclic loading. Engineering Structures, 199.

[37] Krolo P, Grandić D, Smolčić Ž., 2016. Experimental and numerical study of mild steel behaviour under cyclic loading with variable strain ranges. Advances in Materials Science and Engineering, 2016, $1-13$.

[38] Benson, S., D., 2011. Progressive collapse assessment of light weight ship structures. PhD thesis, Newcastle University, UK.

[39] Smith, C.S., Davidson, P.C., Chapman, J.C., Dowling, P.J., 1987. Strength and stiffness of ships' plating under in-plane compression and tension. Transaction of RINA, 130, 277-293. 